\title{
O Método Probabilístico e o Lema Local de Lovász
}

\author{
Iesus Carvalho Diniz
}

\author{
DISSERTAÇÃO APRESENTADA \\ AO INSTITUTO DE MATEMÁTICA E ESTATÍSTICA \\ DA UNIVERSIDADE DE SÃO PAULO \\ PARA OBTENÇÃO \\ DO GRAU DE MESTRE EM ESTATÍSTICA
}

Área de Concentração: Probabilidade

Orientador: Prof. Dr. Fábio Prates Machado

São Paulo, 5 de abril de 2005.

O autor agradece a CAPES pelo apoio financeiro. 


\title{
O Método Probabilístico e o Lema Local de Lovász
}

\author{
Este exemplar corresponde à redação final \\ da dissertação devidamente corrigida \\ e defendida por Iesus Carvalho Diniz \\ e aprovada pela comissão julgadora.
}

São Paulo, 5 de abril de 2005.

Banca examinadora:

Prof. Dr. Fábio Prates Machado (IME-USP)

Prof. Dr. Luis Gustavo Esteves (IME-USP)

Prof. Dr. Oswaldo Scarpa Magalhães Alves (UFG) 
Aos meus pais, Igor e João Carvalho. 


\section{Agradecimentos:}

Agradeço a toda minha família pelo apoio e encorajamento dados a mim. Em especial: meus pais, Igor, João Carvalho; e aos primos Dalmi, Rodrigo, Fábio, Diego, Clara, Rubens, Gabriela, Débora, Micael, Cleuza, Paulo Rogério e Débora Brito.

Agradeço ao meu orientador Prof. Dr. Fábio Prates Machado; pelo profissionalismo, solicitude e amizade dispensados a mim. Ao prof. João Maurício Araújo Mota pela referência moral e intelectual que é. Ao prof. Dalton Francisco de Andrade por tanto incentivar a estudar aqui no IME-USP. Aos profs. Oswaldo Magalhães, Luis Gustavo e Manoel Campelo.

A todos os funcionários e colegas do IME-USP, em especial, Florencia G. Leonardi.

Aos meus conterrâneos de Cedro-Ce e Várzea Alegre-Ce por todos os momentos de descontração e apoio.

A CAPES, pelo suporte financeiro recebido. 


\section{Resumo:}

O Método Probabilístico pode ser entendido como uma ferramenta de ataque a problemas em matemática discreta. A idéia do método é de que na impossibilidade de exibição de estruturas assumindo propriedades desejadas, substituir esta tarefa pela tarefa de montar um espaço probabilístico apropriado, em que o evento reunindo aquelas estruturas tem probabilidade estritamente positiva.

Neste trabalho procuramos mostrar um pouco da essência do Método Probabilistico através de alguns exemplos em combinatória e teoria dos grafos. A partir destes exemplos nota-se a importância do método por sua fácil aplicabilidade e por resolver problemas nos quais nenhuma outra solução é conhecida até hoje.

Dentro das várias técnicas do Método Probabilístico, concentramo-nos no Princípio do Primeiro Momento - baseado no simples fato que se $\mathbb{E}(X) \leq t$ então $\mathbb{P}(X \leq t)>0$ - e no Lema Local de Lovász cujo objeto é o cálculo da probabilidade de não ocorrência simultânea de um grupo de eventos em uma situação em que existe dependência entre estes eventos. Apresentamos a segunda técnica como alternativa em situações em que a aplicação da primeira não surte efeito.

Palavras-chave: Método Probabilístico, Método do Primeiro Momento, Lema Local de Lovász. 


\begin{abstract}
:
The Probabilistic Method can be seen as a tool for attacking problems in discrete mathematics. The basic idea for the method is to construct an appropriate sometimes artificial - probability space showing that an event consisting of structures of interest has positive probability. While the method is not constructive it shows rigorously that those structures exists.

In this work we introduce the Probabilistic Method by showing some of its applications in Combinatorics and Graph Theory. From these examples one can see the power of the method and some surprisingly easy applications.

Among many technics of the Probabilistic Method we concentrate on the First Moment Principle - which is based on the simple fact that if $\mathbb{E}(X) \leq t$ then $\mathbb{P}(X \leq t)>0$ - and in the Lovász Local Lemma whose object is to compute the probability of non simultaneous occurrence of a set of events in a situation of dependence among these events. We present the latter technic as an alternative in situations when the former clearly fails.
\end{abstract}

Keywords: Probabilistic Method, First Moment Principle, Lovász Local Lemma. 


\section{Sumário}

1 O Método Probabilístico 1

1.1 Introdução . . . . . . . . . . . . . . . . . . . 1

1.2 Exemplos de aplicações . . . . . . . . . . . . . . . . . 3

1.2.1 Número de Ramsey . . . . . . . . . . . . . . . . . . . . 4

1.2.2 2-colorimento de um hipergrafo ............ 8

1.2.3 Torneios ...................... 9

1.3 O Método do Primeiro Momento . . . . . . . . . . . . . . . . 11

1.3.1 Aplicações . . . . . . . . . . . . . . . . . . . . 14

2 O Lema Local de Lovász 19

2.1 Introdução . . . . . . . . . . . . . . . . . . . . . . . . 19

2.2 O Lema Local de Lovász - caso geral . . . . . . . . . . . . . . . . . . 21

2.2.1 Condição suficiente para um limite inferior de $\mathrm{R}(\mathrm{k}, 3) \quad \ldots \quad$. . . 27

2.3 O Lema Local de Lovász - caso simétrico. . . . . . . . . . . . . . . . 29

2.3 .1 Aplicações . . . . . . . . . . . . . . . . . . 32

A Apêndice $\quad 42$ 


\section{Lista de Figuras}

1.1 Caminho hamiltoniano em $K_{5} \ldots \ldots \ldots \ldots \ldots \ldots$

1.2 Grafo completo de ordem $6\left(K_{6}\right) \ldots \ldots \ldots \ldots$

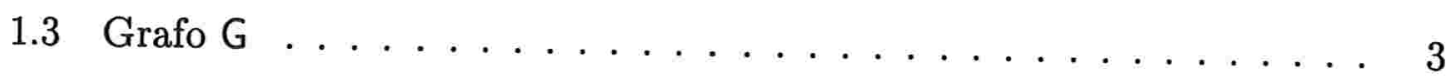

1.4 Grafo complementar $\mathrm{G}^{\prime} \ldots \ldots \ldots \ldots \ldots \ldots$

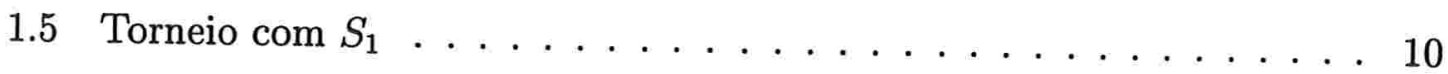

1.6 Torneio $\operatorname{com} S_{2} \ldots \ldots \ldots \ldots \ldots \ldots \ldots \ldots$

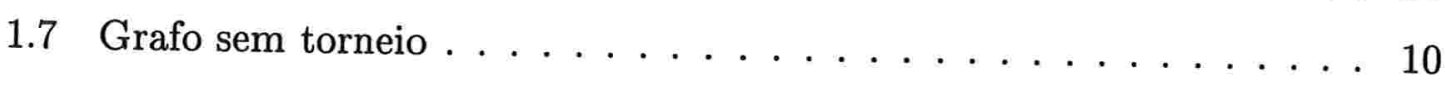

1.8 Torneio sem $S_{2} \ldots \ldots \ldots \ldots \ldots \ldots \ldots \ldots$ 


\section{Capítulo 1}

\section{O Método Probabilístico}

\subsection{Introdução}

O Método Probabilístico pode ser entendido como uma ferramenta de ataque a problemas em matemática discreta. A idéia do método é de que na impossibilidade de exibição de estruturas assumindo propriedades desejadas, substituir esta tarefa por montar um espaço probabilístico apropriado, em que o evento reunindo aquelas estruturas tem probabilidade estritamente positiva.

Para muitos, a primeira utilização do que se entende hoje como Método Probabilístico, está em um trabalho de Szele [9] em 1943. Lá foi apresentado um limite inferior para o número máximo de caminhos sem laços passando por todos os vértices de um grafo completo orientado de ordem $n\left(K_{n}\right)$. Tais caminhos são chamados de hamiltonianos e o grafo que os contém de torneio.

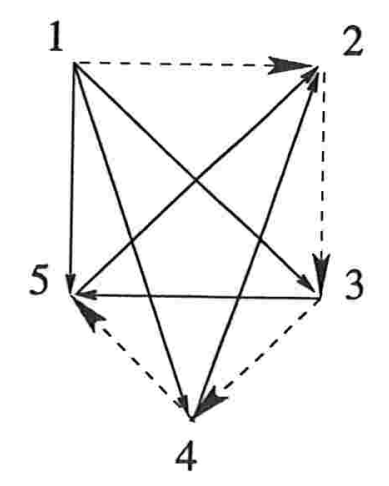

Figura 1.1: Caminho hamiltoniano em $K_{5}$

A seguir, como forma de abordar o que entendemos como a idéia central 
do método, apresentaremos os passos da prova daquele limite inferior em palavras. Inicialmente, Szele observou que cada permutação $\sigma=(\sigma(1), \ldots, \sigma(n))$ dos vértices, poderia ser ou não um caminho hamiltoniano. Seria de fato, caso o torneio em questão tivesse o elo que liga os vértices $\sigma(i)$ e $\sigma(i+1)$, apontado para $\sigma(i+1)$. Isto para todo $i$ de 1 a $n-1$.

A idéia básica seria então considerar torneios (grafos completos orientados) aleatórios uniformes e escrever a variável $X=$ total de caminhos hamiltonianos, como soma de variáveis indicadoras do evento de a permutação ser um caminho hamiltoniano. Para cada uma das $n$ ! permutações, a probabilidade de ser um caminho hamiltoniano para o torneio aleatório é $2^{-(n-1)}$. Logo a esperança da variável $X$ é igual a $n ! 2^{-(n-1)}$ e do fato que uma variável aleatória inteira assume valores maiores ou iguais a sua média deve ter probabilidade estritamente maior do que zero, Szele concluiu que existem torneios de $n$ vértices com pelo menos $n ! \cdot 2^{-(n-1)}$ caminhos hamiltonianos.

Por trás da abordagem de ataque ao problema acima há uma idéia extremamente clara: criar um espaço de probabilidades apropriado para o conjunto das estruturas combinatórias nas quais desejamos verificar a existência de elementos que exibem determinada propriedade. No caso acima, a estrutura são os torneios e a propriedade desejada é a existência de pelo menos $n ! \cdot 2^{-(n-1)}$ caminhos hamiltonianos. Embora sem exibir os torneios que têm aquela propriedade, Szele provou que eles existem, pois, na liguagem probabilística, são eventos de probabilidade positiva.

A partir do ano 1947, esta idéia foi desenvolvida e aplicada com grande talento pelo matemático húngaro Paul Herdôs. Recentemente, através do importante papel da aleatoriedade em áreas como computação teórica, combinatória e teoria dos grafos; este tipo de abordagem tem experimentado intenso desenvolvimento. Para finalizar esta introdução, gostaríamos de salientar que o objetivo deste trabalho é discutir algumas possibilidades de aplicação do método probabilístico em combinatória e teoria dos grafos, e não o de apresentar resultados ótimos ou contemporâneos destas áreas. 


\subsection{Exemplos de aplicações}

O Método Probabilístico não tem como objetivo construir exemplos de estruturas em que são exibidas propriedades desejadas. Trata-se de um método para provar rigorosamente a existência das mesmas. Nesta seç̧ão apresentamos um conjunto de exemplos, dado que consideramos ser esta a melhor forma de apresentação do método. Iniciamos esta tarefa com a apresentação de algumas definições.

Um Grafo $G=(V, E)$ é formado por um conjunto de pontos $V$, chamados vértices e um conjunto de pares de vértices $E$, chamados de elos. Dois vértices formando um elo são chamados de adjacentes. A ordem de um grafo é a cardinalidade de V. Um grafo é dito completo se todos os seus vértices são adjacentes. Denotamos o grafo completo de ordem $n$ por $K_{n}$. A partir de G, definimos o grafo complementar $\mathrm{G}^{\prime}$, que apresenta os mesmos vértices de $\mathrm{G}$ e no qual dois vértices são adjacentes, se e somente se, não o forem em $G$. Definimos também $G_{n, p}$ o grafo aleatório em que cada par de vértices é adjacente com probabilidade $p$, independentemente dos demais elos.

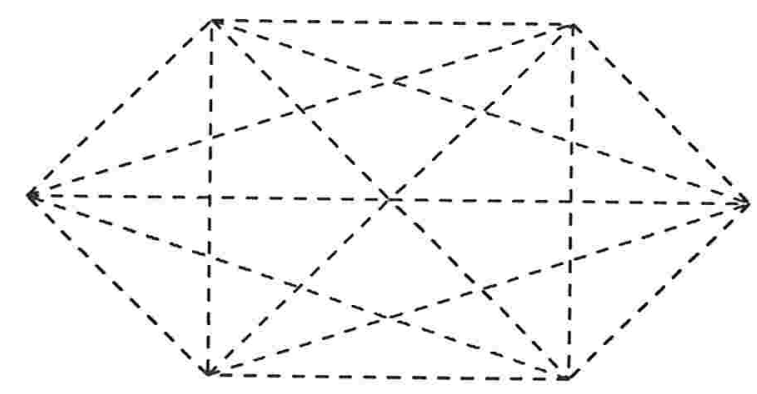

Figura 1.2: Grafo completo de ordem $6\left(K_{6}\right)$

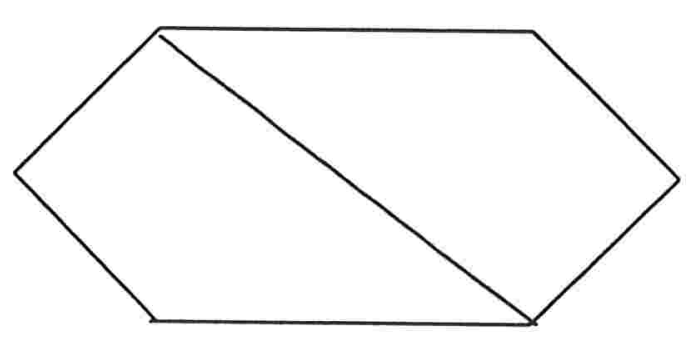

Figura 1.3: Grafo G

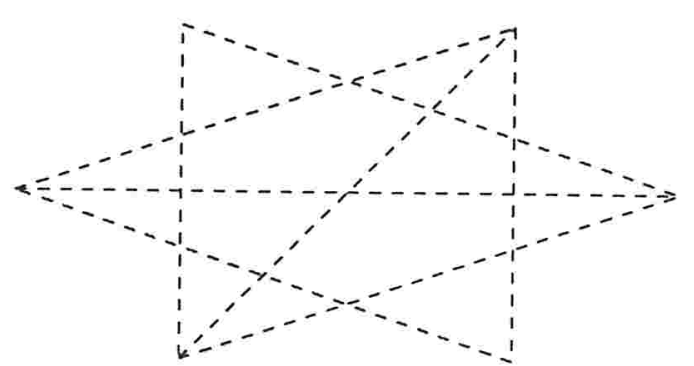

Figura 1.4: Grafo complementar $\mathrm{G}^{\prime}$ 


\subsubsection{Número de Ramsey}

A primeira utilização do que hoje se entende como Método Probabilístico apareceu em um artigo de Szele [9] em 1943, mas é consenso que foi Paul Erdốs, a partir de 1947, que primeiro compreendeu o poder do método, aplicando-o por décadas aos mais variados tipos de problemas. O primeiro de seus artigos a tratar deste método apresenta limites inferiores e superiores para o número de Ramsey, que definimos a seguir.

Definição 1.1 número de Ramsey $R(k, l)$ é o menor inteiro $n$ para o qual, em qualquer colorimento utilizando 2 cores (azul e vermelho) dos elos de um grafo completo de ordem $n$, ou há um grafo completo de ordem $k$ azul, ou há um grafo completo de ordem l vermelho.

A seguir, apresentamos e provamos parte de um teorema de Paul Erdốs em 1947 [2], que estabelece uma cota inferior para o número de Ramsey na forma diagonal $(R(k, k))$, via um argumento de contagem e usando a técnica do Método Probabilístico. O melhor limite superior que fomos capazes de encontrar na literatura é $\left(\begin{array}{c}2 k-2 \\ k-1\end{array}\right)$, cuja obtenção baseia-se em um argumento conhecido como princípio da casa dos pombos. Este limite também é apresentado por Paul Erdôs em 1947 [2].

Teorema 1.1 Se $\left(\begin{array}{l}n \\ k\end{array}\right) 2^{1-\left(\begin{array}{c}k \\ 2\end{array}\right)}<1$ então $R(k, k)>n$. Além disto, para $k \geq 3$ temos que $R(k, k)>2^{\frac{k}{2}}$.

Demonstração: Considere o grafo $K_{k}$ (completo de ordem $k$ ) e o processo aleatório de colorimento de seus elos utilizando duas cores, em que cada elo é colorido independentemente um do outro, de azul ou vermelho, com iguais probabilidade.

Inicialmente observamos que para qualquer conjunto fixado de $k$ vértices e o subgrafo completo que os utiliza, a probabilidade de termos o evento da monocromaticidade é $2^{1-\left(\begin{array}{c}k \\ 2\end{array}\right)}$. Por conta disto, a probabilidade de observarmos a monocromaticidade para algum subgrafo completo de $k$ vértices é, pela propriedade de suba-

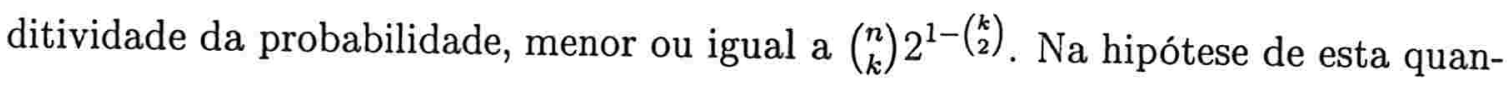
tidade ser estritamente menor do que 1 , temos a existência de colorimentos em que 
nenhum subgrafo completo com $k$ vértices é monocromático. De outra maneira, $R(k, k)>n$.

Para provar a segunda parte do teorema, observemos que o número total de grafos de ordem $n$ contendo o grafo $K_{k}$ (no sentido de conter seus vértices e elos) é:

$$
2^{(k \cdot(n-k))} \cdot 2^{\left(\begin{array}{c}
(n-k) \\
2
\end{array}\right)}=\frac{2^{\left(\begin{array}{c}
n \\
2
\end{array}\right)}}{2^{\left(\begin{array}{c}
k \\
2
\end{array}\right)}}=2^{\left(\begin{array}{c}
n \\
2
\end{array}\right)-\left(\begin{array}{c}
k \\
2
\end{array}\right)}
$$

Para verificar a equação (1.1), observe que existem $k \cdot(n-k)$ possíveis elos ligando os $k$ vértices do grafo $K_{k}$ aos $(n-k)$ vértices adicionais. Além disso, existem $\left(\begin{array}{c}n-k \\ 2\end{array}\right)$ possíveis elos ligando cada par dos $n-k$ vertices adicionais. Cada um destes elos pode aparecer ou não no novo grafo de ordem $n$ contendo o grafo $K_{k}$.

Logo, o número de grafos de ordem $n$, que contém um subgrafo completo de ordem $k$, é inferior ou igual a

$$
\left(\begin{array}{l}
n \\
k
\end{array}\right) \cdot \frac{2^{\left(\begin{array}{c}
n \\
2
\end{array}\right)}}{2^{\left(\begin{array}{c}
k \\
2
\end{array}\right)}}
$$

O número de diferentes grafos com $n$ vértices é $2^{\left(\begin{array}{c}n \\ 2\end{array}\right)}$, já que existem $\left(\begin{array}{l}n \\ 2\end{array}\right)$ possíveis escolhas para cada par de vértices, e, para cada escolha feita, há duas possibilidades (adjacente ou não adjacente), independente das demais.

Acontece que:

$$
\left(\begin{array}{l}
n \\
k
\end{array}\right)<\frac{n^{k}}{k !}
$$

e de (1.2) tem-se que

$$
\left(\begin{array}{l}
n \\
k
\end{array}\right) \cdot \frac{2^{\left(\begin{array}{c}
n \\
2
\end{array}\right)}}{2^{\left(\begin{array}{c}
k \\
2
\end{array}\right)}}<\frac{n^{k}}{k !} \cdot \frac{2^{\frac{n \cdot(n-1)}{2}}}{2^{\frac{k \cdot(k-1)}{2}}}
$$

Se $n \leq 2^{\frac{k}{2}}$ e $k \geq 3$ tem-se que:

$$
\frac{n^{k}}{k ! \cdot 2} \leq \frac{2^{\frac{k}{2}}}{k !(k-1)}<\frac{1}{2}
$$

De (1.4) e (1.3) em (1.1) resulta que:

$$
\left(\begin{array}{l}
n \\
k
\end{array}\right) \cdot \frac{2^{\left(\begin{array}{l}
n \\
2
\end{array}\right)}}{2^{\left(\begin{array}{c}
k \\
2
\end{array}\right)}}<\frac{1}{2} \cdot 2^{\left(\begin{array}{l}
n \\
2
\end{array}\right)}
$$


De (1.5) tem-se que o número total de grafos com $n=2^{\frac{k}{2}}$ vértices contendo um subgrafo completo de ordem $k$ é menor do que $\frac{1}{2} \cdot 2\left(\begin{array}{c}n \\ 2\end{array}\right)$.

Segue-se que o número de grafos que apresentam um subgrafo $K_{k}$ nele ou em

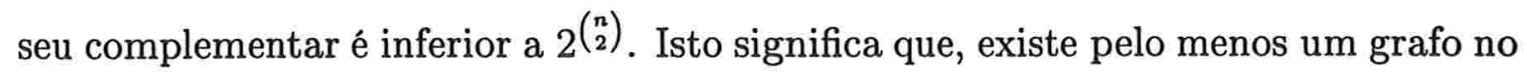
conjunto de todos os $2^{\left(\begin{array}{l}n \\ 2\end{array}\right)}$ grafos possíveis com $n$ vértices em que não encontramos $K_{k}$ tanto nele quanto no seu grafo complementar. Ou seja, $R(k, k)>2^{\frac{k}{2}}$.

Observamos que acima foi feito o uso do Método Probabilístico, simplesmente usando um argumento de contagem. Em muitas situações não temos espaços finitos envolvendo uma específica propriedade. Neste contexto, a probabilidade passa a ser essencial. Algumas técnicas que tratam situações como esta são: Método do Primeiro Momento, Método do Segundo Momento, Lema Local de Lovász, desigualdades envolvendo correlações, desigualdade de Azuma e desigualdade de Talagrand.

A condição envolvendo $n$ e $k$ no teorema anterior é um caso particular da condição mais geral dada a seguir.

Teorema 1.2 Se $\left(\begin{array}{l}n \\ k\end{array}\right) \cdot p^{\left(\begin{array}{l}k \\ 2\end{array}\right)}+\left(\begin{array}{l}n \\ t\end{array}\right) \cdot(1-p)^{\left(\begin{array}{l}t \\ 2\end{array}\right)}<1$ para algum $p \in(0,1)$, então $R(k, t)>$ $n$.

Demonstração: Considere a probabilidade de um elo ser azul $p$ e de ser vermelho $1-p$, e que cada elo é colorido independentemente dos demais. Assuma que:

$$
\omega_{(i, j)}= \begin{cases}1 & \text { se o elo }(i, j) \text { for azul. } \\ 0 & \text { se o elo }(i, j) \text { for vermelho }\end{cases}
$$

Sejam os seguintes conjuntos e eventos aleatórios definidos a seguir.

$\Omega:\{0,1\}^{\left(\begin{array}{l}n \\ 2\end{array}\right)}$ Representa o conjunto de todos os colorimentos de $K_{n}$.

$$
\mathrm{R}_{i}:\left\{i_{1}, i_{2}, \ldots, i_{k}\right\} \subset \mathrm{V} ; \quad\left|\mathrm{R}_{i}\right|=k \quad \forall i \in\left\{1,2, \ldots,\left(\begin{array}{l}
n \\
k
\end{array}\right)\right\} \quad \text { Representa o i-ésimo }
$$
subgrafo completo de ordem $k$, induzido pelos vértices de $\mathrm{R}_{i}$ em $K_{n}$.

$\mathrm{S}_{i}:\left\{i_{1}, i_{2}, \ldots, i_{t}\right\} \subset \mathrm{V} ; \quad\left|\mathrm{S}_{i}\right|=t \quad \forall i \in\left\{1,2, \ldots,\left(\begin{array}{c}n \\ t\end{array}\right)\right\} \quad$ Representa o i-ésimo subgrafo completo de ordem $t$, induzido pelos vértices de $\mathrm{S}_{i}$ em $K_{n}$. 
$\mathrm{E}_{\mathrm{R}_{i}}:\{i, j\} \subset\left\{i_{1}, i_{2}, \ldots, i_{k}\right\}: i<j$ Representa o conjunto dos elos de $\mathrm{R}_{i}$.

$\mathrm{E}_{\mathrm{S}_{i}}:\{i, j\} \subset\left\{i_{1}, i_{2}, \ldots, i_{t}\right\}: i<j$ Representa o conjunto dos elos de $\mathrm{S}_{i}$.

$A_{\mathrm{R}_{i}}:\left\{\omega_{(i, j)}=1 \forall\{i, j\} \subset \mathrm{E}_{\mathrm{R}_{i}}\right\}$ Representa o evento aleatório de que todos os elos de $\mathrm{R}_{i}$ são azuis.

$A_{\mathrm{S}_{i}}:\left\{\omega_{(i, j)}=0 \forall\{i, j\} \subset \mathrm{E}_{\mathrm{S}_{i}}\right\}$ Representa o evento aleatório de que todos os elos de $S_{i}$ são vermelhos.

A partir dos conjuntos e eventos aleatórios definidos:

$$
\begin{aligned}
& \mathbb{P}\left(A_{\mathrm{R}_{i}}\right)=(p)^{\left(\begin{array}{l}
k \\
2
\end{array}\right)} \\
& \mathbb{P}\left(A_{\mathrm{S}_{i}}\right)=(1-p)^{\left(\begin{array}{l}
t \\
2
\end{array}\right)}
\end{aligned}
$$

O evento de que pelo menos um subgrafo completo de ordem $k\left(K_{k}\right)$ ou de ordem $t$ $\left(K_{t}\right)$ de $K_{n}$ seja monocromático é:

$$
A=\bigcup_{i=1}^{\left(\begin{array}{l}
n \\
k
\end{array}\right)} A_{\mathrm{R}_{i}} \cup \bigcup_{i=1}^{\left(\begin{array}{l}
n \\
t
\end{array}\right)} A_{\mathrm{S}_{i}}
$$

De (1.6) tem-se que a probabilidade de que pelo menos um subgrafo completo de ordem $k$ seja azul ou de ordem $t$ seja vermelho é inferior a 1 , pois:

$$
\begin{aligned}
& \mathbb{P}(A)=\mathbb{P}\left(\bigcup_{i=1}^{\left(\begin{array}{c}
n \\
k
\end{array}\right)} A_{\mathrm{R}_{i}} \cup \bigcup_{i=1}^{\left(\begin{array}{c}
n \\
t
\end{array}\right)} A_{S_{i}}\right) \leq \sum_{i=1}^{\left(\begin{array}{c}
n \\
k
\end{array}\right)} \mathbb{P}\left(A_{\mathrm{R}_{i}}\right)+\sum_{i=1}^{\left(\begin{array}{c}
n \\
t
\end{array}\right)} \mathbb{P}\left(A_{\mathrm{S}_{i}}\right)= \\
&\left(\begin{array}{l}
n \\
k
\end{array}\right) \cdot(p)^{\left(\begin{array}{l}
k \\
2
\end{array}\right)}+\left(\begin{array}{l}
n \\
t
\end{array}\right) \cdot(1-p)^{\left(\begin{array}{c}
t \\
2
\end{array}\right)}<1
\end{aligned}
$$

Em que a última desigualdade (1.7) é verdadeira por hipótese.

O evento de que não há nenhum subgrafo completo de ordem $k$ monocromático azul e de ordem $t$ monocromático vermelho, pertencente a $K_{n}$, é:

$$
\bar{A}=\bigcap_{i=1}^{\left(\begin{array}{l}
n \\
k
\end{array}\right)} \bar{A}_{\mathrm{R}_{i}} \cap \bigcap_{i=1}^{\left(\begin{array}{l}
n \\
t
\end{array}\right)} \bar{A}_{\mathrm{S}_{i}}
$$

De (1.7) tem-se que:

$$
1-\mathbb{P}(A)>0 \Rightarrow \mathbb{P}(\bar{A})>0
$$


Mas $\mathbb{P}(\bar{A})>0$ significa que os eventos $\bar{A}_{\mathrm{R}_{i}}$ e $\bar{A}_{\mathrm{S}_{i}}$ ocorrem para todo $i \epsilon$ $\left\{1,2, \ldots,\left(\begin{array}{l}n \\ k\end{array}\right)\right\}$ e para todo $i \in\left\{1,2, \ldots,\left(\begin{array}{c}n \\ t\end{array}\right)\right\}$, o que equivale a dizer que: existe pelo menos um colorimento de $K_{n}$ sem apresentar nenhum subgrafo aleatório completo de ordem $k$ monocromático azul e de ordem $t$ vermelho.

\subsubsection{2-colorimento de um hipergrafo}

Até o momento trabalhamos com o conceito de grafo. Uma generalização natural deste conceito é o hipergrafo. Um hipergrafo é um par $H=(V, E)$, em que $V$ é um conjunto finito de elementos chamados de vértices e $E$ é uma família de subconjuntos de $\mathrm{V}$ (não necessariamente um par) chamados de hiperelos. Um hipergrafo é dito $n$-uniforme se cada um de seus hiperelos possui $n$ vértices. Dizemos que um hipergrafo é 2-colorável, caso seja possível colorir seus vértices utilizando 2 cores de maneira que nenhum hiperelo seja monocromático.

A seguir, apresentamos um resultado de Paul Erdốs que trata do processo de 2-colorimento de hipergrafos. A consequência deste resultado é que o número mínimo de hiperelos que um hipergrafo $n$-uniforme precisa ter para não admitir um 2-colorimento é pelo menos $2^{n-1}$.

Teorema 1.3 Todo hipergrafo $n$-uniforme com menos do que $2^{n-1}$ hiperelos é 2colorável.

Demonstração: Sejam os conjuntos e eventos aleatórios definidos a seguir. Considere o colorimento do hipergrafo em que cada elo tem iguais probabilidades de ser azul ou vermelho.

V: Representa o conjunto de todos os vértices do hipergrafo.

E: Representa o conjunto de todos os hiperelos. Como o hipergrafo é n-uniforme, temos que para todo $e_{i} \in \mathrm{E},\left|e_{i}\right|=n$.

$\Omega$ : É o espaço amostral associado ao colorimento do hipergrafo. 
$E_{i}$ : É o evento aleatório de que o i-ésimo hiperelo de $\mathrm{H}$ é monocromático.

A: É o evento aleatório de que algum hiperelo de $\mathrm{H}$ é monocromático.

Do que foi falado acima temos:

$$
\begin{gathered}
|\mathrm{E}|<2^{(n-1)} \\
A=\bigcup_{i \in \mathrm{E}} E_{i} \\
\mathbb{P}\left(E_{i}\right)=2 \cdot\left(\frac{1}{2}\right)^{n}=2^{-(n-1)}
\end{gathered}
$$

A probabilidade do evento de que pelo menos um hiperelo de $\mathrm{H}$ seja monocromático, é inferior a um, pois:

$$
\mathbb{P}(A)=\mathbb{P}\left(\bigcup_{i \in \mathrm{E}} E_{i}\right) \leq \sum_{i \in \mathrm{E}} \mathbb{P}\left(E_{i}\right)<2^{(n-1)} \cdot 2^{-(n-1)}=1
$$

1. A primeira desigualdade de (1.8) vem da propriedade de subaditividade da probabilidade.

2. A segunda desigualdade de (1.8) é decorrente da cardinalidade de E ser inferior a $2^{(n-1)}$.

De (1.8) tem-se que a probabilidade de que nenhum hiperelo de $\mathrm{H}$ seja monocromático é positiva, pois:

$$
\mathbb{P}(\bar{A})=1-\mathbb{P}(A)>0 .
$$

Acontece que $\mathbb{P}(\bar{A})>0 \Leftrightarrow \bar{A} \neq \varnothing$, ou seja, existe pelo menos um hipergrafo que apresenta todos os seus hiperelos não monocromáticos para algum 2-colorimento.

\subsubsection{Torneios}

Um grafo completo orientado é dito um torneio. Se em tal grafo, atribuirmos uma distribuição de probabilidade à orientação de cada elo, teremos um torneio aleatório. Cada vértice do grafo orientado pode ser pensado como um jogador e um elo de $x$ para $y$ significando que $x$ vence $y$. Um torneio aleatório com $n$ jogadores 
$\left(T_{n}\right)$ é dito ter a propriedade $S_{k}$, se para todo conjunto $K \subset \mathrm{V}$ com $|K|=k$, existe $y \notin K$ e um subgrafo em que todos os elos emanam de $y$ para os vértices de $K$. $\mathrm{O}$ próximo teorema nos dá uma condição suficiente para a existência de um torneio aleatório $\left(T_{n}\right)$ com propriedade $S_{k}$.

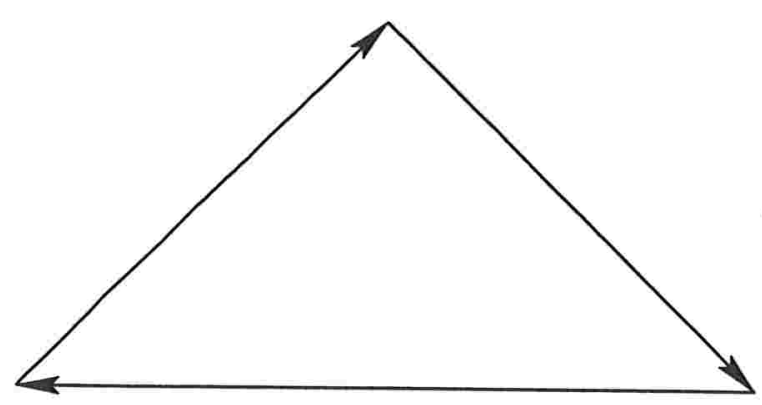

Figura 1.5: Torneio com $S_{1}$

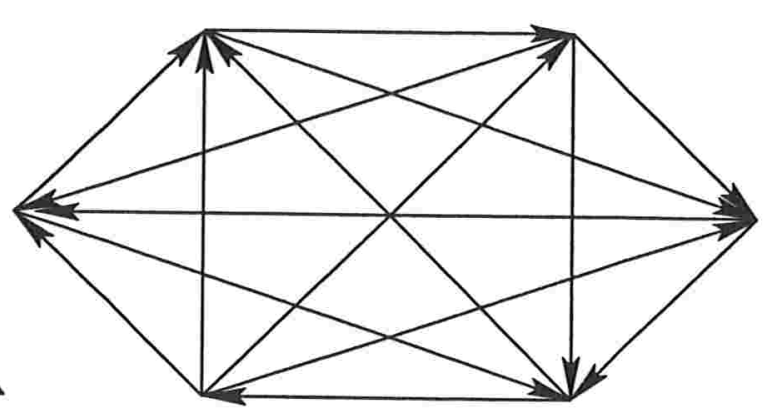

Figura 1.6: Torneio com $S_{2}$

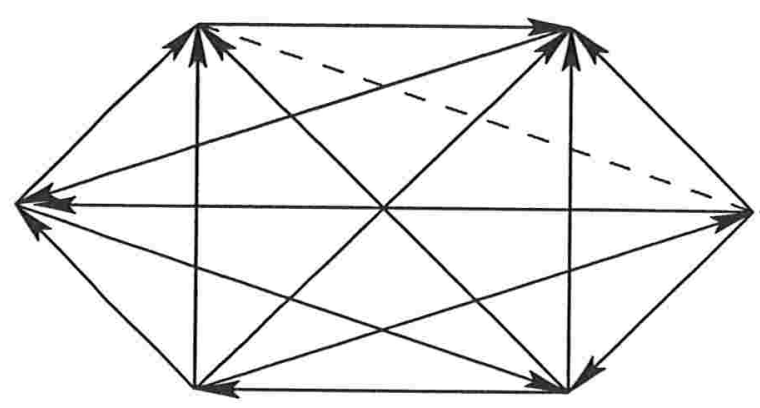

Figura 1.7: Grafo sem torneio

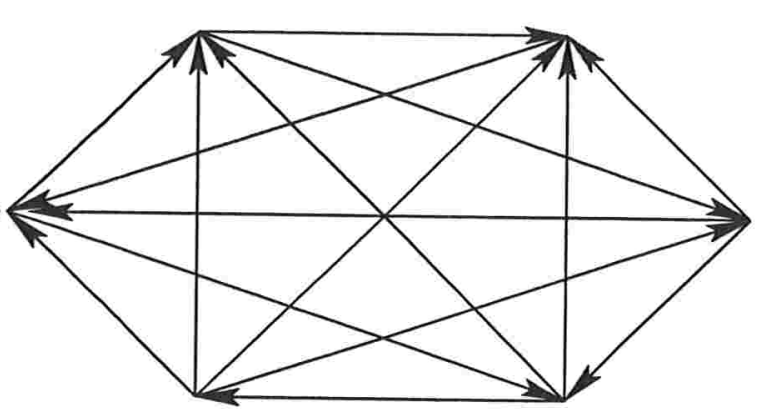

Figura 1.8: Torneio sem $S_{2}$

Teorema 1.4 Para todo $k$ fixado, há um torneio $T_{n}$ com propriedade $S_{k}$.

Demonstração: Considere que cada jogador tem a mesma probabilidade de ganhar ou perder de qualquer outro e que cada partida ocorre independentemente das demais. Para qualquer conjunto $K \subset\{1,2, \ldots, n\}$ tal que $|K|=k$, seja $A_{i}$ o evento de que nenhum jogador pertencente a $K^{c}$ ganha de todos os elementos de $K$. Segue-se que:

$$
\mathbb{P}\left(A_{i}\right)=\left(1-2^{-k}\right)^{n-k}
$$


Logo, o evento de que não haja um torneio com a propriedade $S_{k}$ é,

$$
A=\bigcup_{i=1}^{\left(\begin{array}{l}
n \\
k
\end{array}\right)} A_{i}
$$

cuja proprabilidade pode ser majorada pela subaditividade da probabilidade por:

$$
\mathbb{P}(A) \leq\left(\begin{array}{l}
n \\
k
\end{array}\right) \cdot\left(1-2^{-k}\right)^{n-k}
$$

Se tomarmos n de tal maneira que (1.9) seja menor do que 1, temos uma condição suficiente para que:

$$
A^{c}=\bigcap_{i=1}^{\left(\begin{array}{l}
n \\
k
\end{array}\right)} A_{i}^{c} \neq \varnothing
$$

Esta última equação nos diz que para qualquer grupo de $k$ jogadores, escolhidos de um conjunto finito de tamanho $n=n(k)$, há um outro jogador pertencente aos demais $n-k$ elementos que ganha de todos os $k$ escolhidos inicialmente, implicando que o torneio aleatório tenha a propriedade $S_{k}$.

Nos quatro teoremas acima, vimos o mecanismo geral do Método Probabilístico. Primeiro definimos um espaço de probabilidades em que é definido um critério para a obtenção de todos os objetos a ele pertencente. Define-se um evento que representa as propriedades de interesse que se quer verificar no objeto aleatório e caso este evento seja diferente do vazio, temos que há pelo menos um ponto no conjunto de todos os objetos aleatórios, espaço amostral, que satisfaz as propriedades de interesse que se quer verificar.

\subsection{O Método do Primeiro Momento}

O Método do Primeiro Momento é a ferramenta mais básica do Método Probabilístico. Nos teoremas vistos na seç̧ão anterior (1.2), tínhamos uma condição a partir da qual era possível associar uma probabilidade positiva ao evento de interesse. Esta condição era estabelecida usando a subaditividade da probabilidade ao evento formado pela união de eventos que infringiam alguma característica da estrutura de interesse e fazendo esta probabilidade menor do que 1 . 
Acontece que se soubermos que o valor esperado $(\mathbb{E})$ de uma variável aleatória for menor ou igual que $t$, então a probabilidade de tal variável aleatória ser menor ou igual que $t$ é positiva. O Método do Primeiro Momento usa esse fato para estabelecer uma probabilidade positiva ao objeto aleatório em questão.

Proposição 1.1 Se $\mathbb{E}(X) \leq t$, então $\mathbb{P}(X \leq t)>0$

Demonstração: A demonstração será feita por redução ao absurdo, e para tanto, suporemos falsa a conclusão e chegaremos à negação da hipótese.

$$
\begin{gathered}
\mathbb{P}(X \leq t)=0 \Rightarrow \mathbb{P}(X>t)=1 \\
\mathbb{E}(X)=\int_{-\infty}^{\infty} x d F(x)=\int_{t}^{\infty} x d F(x)>t \int_{t}^{\infty} d F(x)= \\
t \mathbb{P}(X>t)=t \cdot 1=t
\end{gathered}
$$

De (1.11) temos que:

$$
\mathbb{E}(X)>t
$$

Observação 1.1 Fazendo $t=\mathbb{E}(X)$ na proposição 1.1, temos que:

$$
\mathbb{P}(X \leq \mathbb{E}(X))>0 \Leftrightarrow \mathbb{P}(X>\mathbb{E}(X))<1
$$

A utilização do Método do Primeiro Momento faz-se então através da escolha de uma variável que represente uma quantidade de interesse no problema. A partir disto, é obtido o seu valor esperado, e este é então associado a uma probabilidade positiva correspondente ao evento de a variável assumir um valor não inferior ou superior a ele.

Uma importante desigualdade envolvendo uma variável aleatória que assume valores não negativos e o seu valor esperado é a desigualdade de Markov. 
Teorema 1.5 (Desigualdade de Markov) Seja X uma variável aleatória inteira não negativa e $t>0$. Nestas condições:

$$
\mathbb{P}(X \geq t) \leq \frac{\mathbb{E}(X)}{t}
$$

Demonstração: Para todo $t>0$ seja a variável:

$$
\mathbb{I}=\left\{\begin{array}{lll}
1 & \text { se } & X \geq t \\
0 & \text { se } & X<t
\end{array}\right.
$$

Como $X \geq 0$, tem-se que:

$$
\mathbb{I} \leq \frac{X}{t}
$$

Tomando o valor esperado em (1.12), temos o seguinte:

$$
\mathbb{E}(\mathbb{I}) \leq \frac{\mathbb{E}(X)}{t}
$$

Acontece que:

$$
\mathbb{E}(\mathbb{I})=1 \cdot \mathbb{P}(X \geq t)+0 \cdot \mathbb{P}(X<t)=\mathbb{P}(X \geq t)
$$

De (1.14) em (1.13) segue-se que:

$$
\mathbb{P}(X \geq t) \leq \frac{\mathbb{E}(X)}{t}
$$

Observação 1.2 A condição de subaditividade da probabilidade pode ser vista como uma aplicação da desigualdade de Markov.

Demonstração: Sejam $A_{1}, A_{2}, \ldots, A_{n}$ eventos, $X_{i}$ a variável indicadora do i-ésimo evento e $X=\sum_{i=1}^{n} X_{i}$ a variável que estabelece o número de $A_{i^{\prime} s}$ que ocorrem.

$$
\begin{array}{r}
\mathbb{P}\left(\bigcup_{i=1}^{n} A_{i}\right)=\mathbb{P}(X>0)=\mathbb{P}(X \geq 1) \leq \frac{\mathbb{E}(X)}{1}=\mathbb{E}\left(\sum_{i=1}^{n} X_{i}\right) \\
\sum_{i=1}^{n} \mathbb{E}\left(X_{i}\right)=\sum_{i=1}^{n} \mathbb{P}\left(A_{i}\right)
\end{array}
$$




\subsubsection{Aplicações}

A seguir será enunciado um teorema que mostra a aplicação do Método do Primeiro Momento na teoria dos grafos. Será definida uma variável aleatória (inteira) e a partir do cálculo de seu valor esperado é encontrada uma probabilidade positiva de a nossa variável assumir um valor de interesse. A esse valor, que corresponde a um evento diferente do vazio no espaço amostral, associamos o objeto aleatório não contruído explicitamente e que satisfaz as propriedades de interesse.

Teorema 1.6 (4-colorimento de um hipergrafo) $S e n \geq 2$ e $\mathrm{H}=(\mathrm{V}, \mathrm{E})$ é um hipergrafo $n$-uniforme com $|\mathrm{E}|=4^{(n-1)}$ hiperelos, então há um colorimento de $\mathrm{V}$ com 4 cores de forma que nenhum hiperelo é monocromático.

Demonstração: Admita que cada vértice é colorido com uma das quatros cores, independentemente dos demais e com probabilidades iguais a $\frac{1}{4}$. Seja a variável aleatória $X_{e}$ assim definida:

$$
X_{e}= \begin{cases}1 & \text { se o hiperelo } e \text { for monocromático. } \\ 0 & \text { se o hiperelo } e \text { não for monocromático. }\end{cases}
$$

O número de elos monocromáticos é então dado por:

$$
\begin{gathered}
X=\sum_{e \in \mathrm{E}(\mathrm{H})} X_{e} \\
\mathbb{P}\left(X_{e}=1\right)=4 \cdot \frac{1}{4^{n}}=4^{(1-n)}
\end{gathered}
$$

Pela linearidade da esperança tem-se:

$$
\begin{aligned}
& \mathbb{E}(X)=\mathbb{E}\left(\sum_{e \in \mathrm{E}(\mathrm{H})} X_{e}\right)=\sum_{\substack{e \in \mathrm{E}(\mathrm{H}) \\
|\mathrm{E}(\mathrm{H})| \cdot 4^{(1-n)}}} \mathbb{E}\left(X_{e}\right)=\sum_{e \in \mathrm{E}(\mathrm{H})} \mathbb{P}\left(X_{e}=1\right) \leq \\
& 4^{(n-1)} \cdot 4^{(1-n)}=1
\end{aligned}
$$

Como $X$ é uma variável aleatória inteira não negativa e $\mathbb{E}(X)=1$, temos que:

$$
\mathbb{P}(X=0)>0 \Leftrightarrow \mathbb{P}(X=k)>0 \text { para algum } k>1
$$


Acontece que os eventos de termos monocromaticidade nos hiperelos são positivamente correlacionados. Seja então, $k=4^{n-1}$.

$$
\mathbb{P}\left(X=4^{n-1}\right)>\prod_{i=1}^{4^{n-1}} \mathbb{P}\left(X_{e_{i}}=1\right)=\left(4^{1-n}\right)^{4^{n-1}}
$$

Das equações (1.16) e (1.17) temos que $\mathbb{P}(X=0)>0$

Duas quantidades de interesses em um grafo G são: o número cromático, $\chi(G)$, que é o menor número de cores que podemos atribuir aos vértices de um grafo, de forma que dois vértices adjacentes não recebam a mesma cor; e o número clique; $\omega(\mathrm{G})$, que é a ordem do maior subgrafo completo contido em G.

Observação $1.3 \chi(\mathrm{G}) \geq \omega(\mathrm{G})$ Isto segue do fato de termos $\omega(\mathrm{G})$ cores necessárias ao colorimento do clique, e mais cores diferentes das contidas no clique, se houver algum vértice do clique adjacente a outro vértice fora do clique.

O próximo teorema nos mostra que é possível haver grafos com $\chi$ arbitrariamente grande e $\omega \leq 2$. Tais configurações são possíveis porque existem grafos "livre de triângulos" $(\omega \leq 2)$. A existência de tais grafos é mostrada através do Método do Primeiro Momento.

Teorema 1.7 Para qualquer $k \geq 1$, existe um grafo sem exatamente três vértices adjacentes (livre de triângulos), com número cromático maior do que $k$.

Demonstração: Seja o grafo aleatório $\mathrm{G}_{n, p}$ definido na secção 1.2 , com $p=n^{\frac{-2}{3}}$. Para provarmos que $\chi(G)>k$ é suficiente mostrarmos que não há nenhum conjunto de vértices monocromático cuja cardinalidade seja igual a $\frac{n}{k}$, ou seja, se $S_{j}$ for o conjunto estável que recebe a cor $j$, tem-se $\left|S_{j}\right|<\left\lceil\frac{n}{k}\right\rceil$. Quando é feito um colorimento nos vértices de um grafo $G$, obedecendo ao critério de dois vértices adjacentes não receberem a mesma cor, o conjunto formado pelos vértices que recebem uma determinada cor, forma um conjunto de vértices independente de cada um dos demais grupos de outras cores, e portanto, cada conjunto de vértices de uma mesma cor são todos não adjacentes. Cada subgrafo formado por vértices de uma mesma cor 
é denominado de conjunto estável ou conjunto independente. O número cromático do grafo $G, \chi(G)$ é, dessa forma, o número de elementos que compõem a menor partição de conjuntos estáveis em que os vértices de $\mathrm{G}$ podem ser particionados.

Seja II o número de conjuntos estáveis de tamanho $\left\lceil\frac{n}{2 k}\right\rceil$. Para cada subconjunto $S$ dos vértices de tamanho $\left\lceil\frac{n}{2 k}\right\rceil$ defina a variável indicadora da seguinte forma:

$$
\begin{gathered}
\mathbb{I}_{S}= \begin{cases}1 & \text { se S é um conjunto estável. } \\
0 & \text { se S não é um conjunto estável. }\end{cases} \\
\mathbb{E}\left(\mathbb{I}_{S}\right)=\mathbb{P}\left(\mathbb{I}_{S}=1\right)=(1-p)^{\left(\left\lceil\frac{n}{2 k}\right]\right)} \\
\mathbb{I}=\sum_{S \subset \vee,|S|=\left\lceil\frac{n}{2 k}\right\rceil} \mathbb{I}_{S}
\end{gathered}
$$

Pela linearidade da esperança:

$$
\begin{array}{r}
\mathbb{E}(\mathbb{I})=\sum_{S \subset \mathrm{V},|S|=\left\lceil\frac{n}{2 k}\right\rceil} \mathbb{E}\left(\mathbb{I}_{S}\right)=\left(\begin{array}{c}
n \\
\left\lceil\frac{n}{2 k}\right\rceil
\end{array}\right) \cdot(1-p)^{\left(\frac{\left.\Gamma \frac{n}{2 k}\right\rceil}{2}\right)}< \\
\left(\begin{array}{c}
n \\
\left\lceil\frac{n}{2 k}\right\rceil
\end{array}\right) \cdot(1-p)^{\left(\frac{n}{2 k}\right)}
\end{array}
$$

Se $x>0$, então $(1-x)<\exp (-x)(\star)$.

Seja a seguinte desigualdade:

$$
\left(\begin{array}{c}
n \\
\left\lceil\frac{n}{2 k}\right\rceil
\end{array}\right)<2^{n}
$$

Pois o número total de subconjuntos de um dado conjunto de tamanho $n$ é maior que o número total daqueles subconjuntos que apresentam tamanho específico de $\left(\begin{array}{c}n \\ {\left[\frac{n}{2 k}\right.}\end{array}\right)$.

$$
\left(\begin{array}{c}
\frac{n}{2 k} \\
2
\end{array}\right)=\frac{\left(\frac{n}{2 k}\right) !}{2 ! \cdot\left(\frac{n}{2 k}-2\right) !}=\frac{\frac{n}{2 k} \cdot\left(\frac{n}{2 k}-1\right) !}{2 ! \cdot\left(\frac{n-4 k}{2 k}\right) !}=\frac{\frac{n}{2 k} \cdot\left(\frac{n}{2 k}-1\right)}{2 !}=\frac{n \cdot(n-2 k)}{8 k^{2}}
$$

De $(1.19)(1.20)$ e $(\star)$ em $(1.18)$, tem-se que:

$$
\mathbb{E}(\mathbb{I})<2^{n} \cdot \exp \left(\frac{-p \cdot n \cdot(n-2 k)}{8 k^{2}}\right)
$$

Sejam as duas seguintes condições: 

1. $(n-2 \cdot k)>\frac{n}{2}$
2. $\quad p=n^{\frac{-2}{3}}$

As duas condições acima em (1.21) nos dão que:

$$
\mathbb{E}(\mathbb{I})<2^{n} \cdot \exp \left(\frac{-n^{\frac{4}{3}}}{16 \cdot k^{2}}\right)
$$

Seja $n$ tal que (1.22) fique majorado por $\frac{1}{2}$. É suficiente impormos a seguinte condição:

$$
\frac{n^{\frac{4}{3}}}{16 \cdot k^{2}} \geq 2 \cdot n
$$

De (1.23) segue-se que:

$$
n \geq 2^{15} \cdot k^{6}
$$

Se tomarmos $n$ como o dado em (1.24) teremos que a condição do item (1) estará satisfeita e, portanto,

$$
\mathbb{E}(\mathbb{I})<\frac{1}{2}
$$

Tem-se portanto que:

$$
\mathbb{P}(\mathbb{I}>0)=\mathbb{P}(\mathbb{I} \geq 1) \leq \frac{\mathbb{E}(\mathbb{I})}{1}<\frac{1}{2}
$$

Seja agora a variável aleatória $T$ que designa o número de subgrafos completos de ordem 3 (triângulos) em $\mathrm{G}_{n, p}$.

$$
\mathbb{E}(T)=\left(\begin{array}{l}
n \\
3
\end{array}\right) \cdot p^{3}<\frac{n^{3}}{3 !} \cdot\left(n^{\frac{-2}{3}}\right)^{3}=\frac{n}{6}
$$

Pela Desigualdade de Markov temos:

$$
\mathbb{P}\left(T \geq \frac{n}{2}\right)<\frac{1}{3}
$$

De (1.25) e (1.26) temos:

$$
\mathbb{P}\left(\{\mathbb{I} \geq 1\} \cup\left\{\mathbb{T} \geq \frac{n}{2}\right\}\right) \leq \mathbb{P}(\{\mathbb{I} \geq 1\})+\mathbb{P}\left(\left\{\mathbb{T} \geq \frac{n}{2}\right\}\right)<1
$$

Acontece que os seguintes eventos são equivalentes:

$$
\left\{\{\mathbb{I} \geq 1\} \cup\left\{\mathbb{T} \geq \frac{n}{2}\right\}\right\}^{c}=\left\{\{\mathbb{I} \geq 1\}^{c} \cap\left\{\mathbb{T} \geq \frac{n}{2}\right\}^{c}\right\}
$$


Logo, de (1.27) e (1.28)

$$
\mathbb{P}\left(\left\{\{\mathbb{I} \geq 1\}^{c} \cap\left\{\mathbb{T} \geq \frac{n}{2}\right\}^{c}\right\}\right)>0
$$

Desde que II e $\mathbb{T}$ são variáveis aleatórias inteiras, (1.29) é equivalente a:

$$
\mathbb{P}\left(\left\{\{\mathbb{I}=0\} \cap\left\{\mathbb{T}<\frac{n}{2}\right\}\right\}\right)>0
$$

Da desigualdade (1.30), temos que há um (pelo menos um) grafo que satisfaz as duas condições estabelecidas na intersecção dos eventos complementares, ou seja, não apresenta conjuntos estáveis de tamanho $\left\lceil\frac{n}{2 k}\right\rceil$ e $|T|<\frac{n}{2}$.

Seja então o seguinte procedimento aplicado aos vértices de um grafo $G$ que satisfaz as duas condições anteriores:

1. Escolha um conjunto de no máximo $\frac{n}{2}$ vértices, em que tais vértices são extraídos dos triângulos em $\mathrm{G}$, de forma que a cada triângulo seja extraído pelo menos um vértice.

2. Seja $\mathrm{V}^{\star}$ o conjunto formado pelos vértices de cada um dos triângulos obedecendo ao procedimento anterior. Seja $G^{\prime}$ o grafo que se obtém de $G$ sem considerar os vértices de $\mathrm{V}^{*}$.

Do item (1) temos que $\left|G^{\prime}\right| \geq \frac{n}{2}$ e do item (2) segue-se que $G^{\prime}$ não apresenta triângulos. Além disso, como $G^{\prime}$ é formado a partir das condições definidas pelos eventos em (1.28), segue-se que ele não apresenta conjuntos estáveis de tamanho $\left\lceil\frac{n}{2 k}\right\rceil \leq\left\lceil\frac{\left|\mathrm{G}^{\prime}\right|}{k}\right\rceil, \log 0 \chi\left(\mathrm{G}^{\prime}\right)>k$. 


\section{Capítulo 2}

\section{O Lema Local de Lovász}

\subsection{Introdução}

Há muitas situações em que o Método do Primeiro Momento não pode ser usado. A essência do Método consiste basicamente em estabelecer uma probabilidade positiva em relação a um valor de interesse da variável aleatória, a partir da comparação com o seu valor esperado. Caso este valor esperado não seja relevante ao cálculo de tal probabilidade, temos que procurar outra alternativa para mostrar que o evento de interesse tem probabilidade estritamente positiva.

Considere, por exemplo, o teorema 1.3, se houver uma quantidade superior a $2^{n-1}$ hiperelos, o Método do Primeiro Momento claramente não se adequa. Assim, entra em cena uma ferramenta mais geral dentro do Método Probabilístico, o Lema Local de Lovász.

Uma das ferramentas que mais servem de suporte à aplicação do Método Probabilístico é o Lema Local de Lovász. Dos exemplos anteriores, ao ser feito uso do Método Probabilístico, era definido um evento ( $A$ no teorema 1.4 ou 1.3) em termos da união de outros eventos $\left(A_{i}\right.$ ou $\left.E_{i}\right)$ que infringiam alguma condição de ocorrência da estrutura de interesse, esta era representada pelo evento complementar $(\bar{A})$. Se o evento $\bar{A}$ estiver associado a uma probabilidade positiva, teremos pelo menos um ponto no espaço amostral que garante a existência de nosso objeto aleatório.

Deparamo-nos, então, com o problema de se determinar a probabilidade de um particular evento de interesse, que é representado pela intersecção dos comple- 
mentares de cada um dos eventos que violam uma particular propriedade do nosso objeto aleatório. O Lema Local de Lovász é uma importante ferramenta para se determinar $\mathbb{P}(\bar{A})$, principalmente, quando a dependência entre os $A_{i}$ é pequena; ou seja, cada evento depende de alguns poucos outros eventos e é independente dos demais.

Seja $A_{i}, i \in\{1,2, \ldots, n\}$, o evento que está associado à condição de a i-ésima restrição da nossa estrutura de interesse ser violada, $p_{i}=\mathbb{P}\left(A_{i}\right)$ e $p=\sup p_{i}$. Os eventos $A_{i}$ serão chamados de eventos "ruins". A questão de interesse passaria a ser então mostrar que:

$$
\mathbb{P}\left(\bigcap_{i=1}^{n} \bar{A}_{i}\right)>0
$$

Em algumas situações é fácil determinar se (2.1) é satisfeita.

1. Se todos os $A_{i}$ são independentes, então:

$$
\mathbb{P}\left(\bigcap_{i=1}^{n} \overline{A_{i}}\right)=\prod_{i=1}^{n} \mathbb{P}\left(\overline{A_{i}}\right) \geq(1-p)^{n}
$$

2. Se $n \cdot p<1$, então:

$$
\mathbb{P}\left(\bigcap_{i=1}^{n} \overline{A_{i}}\right)=\mathbb{P}\left(\bigcup_{i=1}^{n} A_{i}\right)^{c}=1-\mathbb{P}\left(\bigcup_{i=1}^{n} A_{i}\right) \geq 1-\sum_{i=1}^{n} \mathbb{P}\left(A_{i}\right) \geq 1-n \cdot p>0
$$

3. Há $d$ independentes grupos de eventos que podem ser dependentes intragrupos, mas que são independentes intergrupos.

$$
\mathbb{P}\left(\bigcap_{i=1}^{n} \overline{A_{i}}\right) \geq \prod_{i=1}^{\frac{n}{d}}(1-d \cdot p)=(1-d \cdot p)^{\frac{n}{d}}
$$

O que foi feito nos exemplos anteriores envolvendo o Método Probabilístico, consitia em se determinar o conjunto dos eventos "ruins" $\left(A_{i}\right)$, e a partir disto, impor uma condição suficiente obtida da propriedade de subaditividade da probabilidade, da forma $n \cdot p<1$, o que implicaria a não ocorrência de nenhum dos $\left(A_{i}\right)$ com probabilidade positiva. 
Acontece que em muitas situações deixa de ser razoável a técnica acima utilizada. Por exemplo, pode-se ter $n \cdot p>1$ e $\mathbb{P}\left(\bigcap_{i=1}^{n} \overline{A_{i}}\right) \geq(1-p)^{n}>0$, bastando para tanto que os $A_{i}$ sejam independentes.

O Lema Local de Lovász estabelece uma cota inferior para (2.1) e é usado em situações em que nenhuma outra ferramenta pode ser usada. Quando existir uma estrutura de dependência entre os eventos das duas seguintes formas, estamos nas condições de uso do Lema Local de Lovász.

1. Cada evento é independente da maioria dos outros eventos.

2. A probabilidade de nenhum evento "ruim" acontecer for muito pequena.

A partir disso, pode-se mostrar através do Método Probabilístico; com o uso do Lema Local de Lovász, que determinada estrutura pode ser verificada em um espaço de probabilidades adequado com as condições de interesse, desde que este último garante uma probabilidade positiva ao evento em questão.

\subsection{O Lema Local de Lovász - caso geral}

Definição 2.1 (Grafo de Dependência) Um Grafo de Dependência $G=(\mathrm{V}, \mathrm{E})$ associado aos eventos $A_{1}, A_{2}, \ldots, A_{n}$ é tal que:

1. cada evento $A_{i} ; i \in\{1,2, \ldots, n\}$ representa um vértice.

2. Se $A_{i}$ e $A_{j}$ não são independentes, há um elo os unindo.

3. $A_{i}$ é mutuamente independente de todos os eventos $\left\{A_{j}:(i, j) \notin \mathrm{E}\right\}$.

\section{Lema 2.1 (O Lema Local de Lovász - caso geral)}

Sejam $A_{1}, A_{2}, \ldots, A_{n}$ eventos em um espaço de probabilidade qualquer. Para todo $i \in\{1,2, \ldots, n\}$, seja $\mathrm{E}_{i}{ }^{c}=\left\{j ; A_{j}\right.$ é mutuamente independente de $\left.A_{i}\right\}$, ou seja, o conjunto de eventos aleatórios que representam no grafo de dependência os pontos não adjacentes ao vértice correspondente ao evento $A_{i}$. Suponha que existam números reais $x_{1}, x_{2}, \ldots, x_{n}$ satisfazendo as seguintes hipóteses: 


$$
\begin{aligned}
0 & \leq x_{i}<1 \\
\mathbb{P}\left(A_{i}\right) & \leq x_{i} \prod_{(i, j) \in E}\left(1-x_{j}\right) \quad \forall i: 1 \leq i \leq n
\end{aligned}
$$

Se as hipóteses (2.2) e (2.3) forem verificadas, então:

$$
\mathbb{P}\left(\bigcap_{i=1}^{n} \overline{A_{i}}\right) \geq \prod_{i=1}^{n}\left(1-x_{i}\right)
$$

Demonstração: A demonstração será feita por indução no número de vértices de um subconjunto $S$ dos eventos aleatórios associados ao grafo de dependência. Para tanto seja a afirmação a seguir:

Afirmação 2.1 Seja $S \subset\{1,2, \ldots, n\},|S|<n$ com $i \notin S$.

$$
\mathbb{P}\left(A_{i} \mid \bigcap_{j \in S} \bar{A}_{j}\right) \leq x_{i}
$$

1. A probabilidade condicional anterior (2.5) diz que dado um subconjunto qualquer $S \subset\{1,2, \ldots, n\}$, e um evento $A_{i}$, em que $i \notin S$, ou seja, $i$ não pertence ao conjunto de índices ao qual $A_{i}$ está condicionado, a desigualdade é verificada.

2. Admita como hipótese de indução que (2.5) vale para todo $S^{*} \subset S$.

Sejam os conjuntos:

$$
\begin{aligned}
& S_{1}=\{j \in S ;(i, j) \in E\} \\
& S_{2}=\{j \in S ;(i, j) \notin E\}
\end{aligned}
$$

de forma que para um $i \in\{1,2, \ldots, n\}$, tem-se que $A_{i}$ é mutuamente independente dos elementos $\left\{j \in S ; A_{j} \in S_{2}\right\}$.

Sejam os três casos a seguir:

- $S=\varnothing$ 
Se $S=$ vazio, então $\mathbb{P}\left(A_{i} \mid \bigcap_{j \in S} \bar{A}_{j}\right)$ é igual a $\mathbb{P}\left(A_{i}\right)$, que por hipótese (2.3), é menor ou igual a $x_{i} \prod_{(i, j) \in E}\left(1-x_{j}\right)$, e como $0 \leq x_{j}<1$, tem-se que $0<\left(1-x_{j}\right) \leq 1$, portanto $\prod_{(i, j) \in E}\left(1-x_{j}\right) \leq 1$.

Assim,

$$
\mathbb{P}\left(A_{i} \mid \bigcap_{j \in S} \bar{A}_{j}\right)=\mathbb{P}\left(A_{i}\right) \leq x_{i} \prod_{(i, j) \in E}\left(1-x_{j}\right) \leq x_{i}
$$

- $S_{1}=\varnothing$

Neste caso, tem-se que $S=S_{2}$, portanto $\mathbb{P}\left(A_{i} \mid \bigcap_{j \in S} \bar{A}_{j}\right)$ é igual a $\mathbb{P}\left(A_{i} \mid \bigcap_{j \in S_{2}} \bar{A}_{j}\right)$. Por $S_{2}$ ser composto de eventos que são mutuamente independentes de $A_{i}$ (grafo de dependência 2.1), tem-se portanto que $\mathbb{P}\left(A_{i} \mid \bigcap_{j \in S_{2}} \bar{A}_{j}\right)=\mathbb{P}\left(A_{i}\right)$, que é menor ou igual a $x_{i} \prod_{(i, j) \in E}\left(1-x_{j}\right)$, pela hipótese (2.3), em que este último termo é menor ou igual a $x_{i}$.

- $S_{1} \neq \varnothing$ e $S_{2} \neq \varnothing$

$S_{1}=\{j \in S ;(i, j) \in E\}=\left\{j_{1}, j_{2}, \ldots, j_{r}\right\}$

$S_{2}=\{j \in S ;(i, j) \notin E\}$

Tem-se:

$$
\mathbb{P}\left(A_{i} \mid \bigcap_{j \in S} \bar{A}_{j}\right)=\mathbb{P}\left(A_{i} \mid \bigcap_{j \in S_{1}} \bar{A}_{j} \bigcap_{l \in S_{2}} \bar{A}_{l}\right)=\frac{\mathbb{P}\left(A_{i} \bigcap_{j \in S_{1}} \bar{A}_{j} \mid \bigcap_{l \in S_{2}} \bar{A}_{l}\right)}{\mathbb{P}\left(\bigcap_{j \in S_{1}} \bar{A}_{j} \mid \bigcap_{l \in S_{2}} \bar{A}_{l}\right)}
$$

\section{Limitante superior para o numerador:}

$$
\mathbb{P}\left(A_{i} \bigcap_{j \in S_{1}} \bar{A}_{j} \mid \bigcap_{l \in S_{2}} \bar{A}_{l}\right) \leq \mathbb{P}\left(A_{i} \mid \bigcap_{l \in S_{2}} \bar{A}_{l}\right)=\mathbb{P}\left(A_{i}\right) \leq x_{i} \prod_{(i, j) \in E}\left(1-x_{j}\right)
$$

1. A primeira desigualdade é devida à relação de inclusão seguinte.

$$
\left(A_{i} \bigcap_{j \in S_{1}} \bar{A}_{j}\right) \subseteq A_{i}
$$


2. A igualdade se deve ao fato de $A_{i}$ ser mutuamente independente dos elementos pertencentes a $S_{2}$.

3. A segunda desigualdade é verdadeira por hipótese (2.3)'

limitante inferior do denominador:

$$
\begin{gathered}
\mathbb{P}\left(\bigcap_{j \in S_{1}} \bar{A}_{j} \mid \bigcap_{l \in S_{2}} \bar{A}_{l}\right)=\mathbb{P}\left(\bar{A}_{j_{1}} \cap \bar{A}_{j_{2}} \ldots \cap \bar{A}_{j_{r}} \mid \bigcap_{l \in S_{2}} \bar{A}_{l}\right)= \\
\mathbb{P}\left(\bar{A}_{j_{1}} \mid \bigcap_{l \in S_{2}} \bar{A}_{l}\right) \times \mathbb{P}\left(\bar{A}_{j_{2}} \mid \bar{A}_{j_{1}} \bigcap_{l \in S_{2}} \bar{A}_{l}\right) \times \ldots \times \mathbb{P}\left(\bar{A}_{j_{r}} \mid \bar{A}_{j_{1}} \bar{A}_{j_{2}} \ldots \bar{A}_{j_{r-1}} \bigcap_{l \in S_{2}} \bar{A}_{l}\right)= \\
{\left[\begin{array}{l}
\left.1-\mathbb{P}\left(A_{j_{1}} \mid \bigcap_{l \in S_{2}} \bar{A}_{l}\right)\right] \times\left[1-\mathbb{P}\left(A_{j_{2}} \mid \bar{A}_{j_{1}} \bigcap_{l \in S_{2}} \bar{A}_{l}\right)\right] \times \ldots \\
\ldots \times\left[1-\mathbb{P}\left(A_{j_{r}} \mid \bar{A}_{j_{1}} \bar{A}_{j_{2}} \ldots \bar{A}_{j_{r-1}} \bigcap_{l \in S_{2}} \bar{A}_{l}\right)\right]
\end{array}\right.}
\end{gathered}
$$

Usando a hipótese de indução, item (2) na página (22), tem-se que:

$$
\mathbb{P}\left(A_{i} \mid \bigcap_{j \in S^{*}} \bar{A}_{j}\right) \leq x_{i} \quad \text { em que } S^{*} \subset S, \quad S^{*} \neq S \quad \text { e } \quad i \notin S^{*}
$$

Tem-se que:

$$
\begin{gathered}
\mathbb{P}\left(A_{j_{1}} \mid \bigcap_{l \in S_{2}} \bar{A}_{l}\right) \leq x_{j_{1}} \\
\mathbb{P}\left(A_{j_{2}} \mid \bar{A}_{j_{1}} \bigcap_{l \in S_{2}} \bar{A}_{l}\right) \leq x_{j_{2}} \\
\vdots \\
\mathbb{P}\left(A_{j_{r}} \mid \bar{A}_{j_{1}} \bar{A}_{j_{2}} \ldots \bar{A}_{j_{r-1}} \bigcap_{l \in S_{2}} \bar{A}_{l}\right) \leq x_{j_{r}}
\end{gathered}
$$

Assim,

$$
\mathbb{P}\left(\bigcap_{j \in S_{1}} \bar{A}_{j} \mid \bigcap_{l \in S_{2}} \bar{A}_{l}\right)=\mathbb{P}\left(\bar{A}_{j_{1}} \cap \bar{A}_{j_{2}} \cap \ldots \cap \bar{A}_{j_{r}} \mid \bigcap_{l \in S_{2}} \bar{A}_{l}\right)=
$$




$$
\begin{gathered}
\mathbb{P}\left(\bar{A}_{j_{1}} \mid \bigcap_{l \in S_{2}} \bar{A}_{l}\right) \times \mathbb{P}\left(\bar{A}_{j_{2}} \mid \bar{A}_{j_{1}} \bigcap_{l \in S_{2}} \bar{A}_{l}\right) \times \ldots \times P\left(\bar{A}_{j_{r}} \mid \bar{A}_{j_{1}} \bar{A}_{j_{2}} \ldots \bar{A}_{j_{r-1}} \bigcap_{l \in S_{2}} \bar{A}_{l}\right)= \\
{\left[1-\mathbb{P}\left(A_{j_{1}} \mid \bigcap_{l \in S_{2}} \bar{A}_{l}\right)\right] \times\left[1-\mathbb{P}\left(A_{j_{2}} \mid \bar{A}_{j_{1}} \bigcap_{l \in S_{2}} \bar{A}_{l}\right)\right] \times \ldots} \\
\ldots \times\left[1-\mathbb{P}\left(A_{j_{r}} \mid \bar{A}_{j_{1}} \bar{A}_{j_{2}} \ldots \bar{A}_{j_{r-1}} \bigcap_{l \in S_{2}} \bar{A}_{l}\right)\right] \geq \\
{\left[1-x_{j_{1}}\right] \times\left[1-x_{j_{2}}\right] \times \ldots \times\left[1-x_{j_{r}}\right]=} \\
\prod_{j \in S_{1}}\left(1-x_{j}\right) \geq \prod_{(i, j) \in E}\left(1-x_{j}\right)
\end{gathered}
$$

em que a última desigualdade se deve ao fato de $\left|S_{1}\right| \leq|\{j:(i, j) \in E\}|$.

Portanto tem-se de (2.10) e (2.11) em (2.9) que:

$$
P\left(A_{i} \mid \bigcap_{j \in S} \bar{A}_{j}\right) \leq x_{i}
$$

Provada a afirmação, via indução no conjunto $S$, segue-se que:

$$
\begin{array}{r}
\mathbb{P}\left(\bigcap_{i=1}^{n} \bar{A}_{i}\right)=\mathbb{P}\left(\bar{A}_{1}\right) \times \mathbb{P}\left(\bar{A}_{2} \mid \bar{A}_{1}\right) \times \ldots \times \mathbb{P}\left(\bar{A}_{n} \mid \bigcap_{i=1}^{n-1} \bar{A}_{i}\right)= \\
\left(1-\mathbb{P}\left(A_{1}\right)\right) \times\left(1-\mathbb{P}\left(A_{2} \mid \bar{A}_{1}\right)\right) \times \ldots \times\left(\left(1-\mathbb{P}\left(A_{n} \mid \bigcap_{i=1}^{n-1} \bar{A}_{i}\right)\right) \geq\right. \\
\left(1-x_{1}\right) \times\left(1-x_{2}\right) \times \ldots \times\left(1-x_{n}\right)=\prod_{i=1}^{n}\left(1-x_{i}\right) .
\end{array}
$$




\section{Observações do Lema Local de Lovász - caso geral}

Observação 2.1 Como hipóteses do Lema Local de Lovász (2.1), temos que, para cada $i \in\{1,2, \ldots, n\}$, o evento $A_{i}$ é mutuamente independente de todos os eventos $\left\{A_{j}:(i, j) \notin \mathrm{E}\right\}$ e que, para cada $A_{i}$, nessas condições tínhamos a hipótese (2.3) de que:

$$
\mathbb{P}\left(A_{i}\right) \leq x_{i} \prod_{(i, j) \in E}\left(1-x_{j}\right)
$$

Observação 2.2 (Hipótese menos restrita) Seja um subconjunto qualquer dos vértices que representam os eventos aleatórios no grafo de dependência definido da seguinte forma:

$$
S_{2}^{*}=\{j: j \in\{1,2, \ldots, n\} e(i, j) \notin \mathrm{E}\}
$$

Pode-se substituir a condição mais restritiva dada em (2.3) de que cada $A_{i}$ ser mutuamente independente de todos os demais elementos não adjacentes a ele no grafo de dependência em uma hipótese mais geral: em que para todo $A_{i}$, um novo conjunto é formado por qualquer subconjunto de eventos aleatórios que são mutuamente independentes de $A_{i}$. Ou seja, a condição de independência mútua de $A_{i}$, inicialmente posta no Lema Local de Lovász (2.1), com todos os eventos aletórios não adjacentes a ele no grafo de dependência, pode ser substituída por uma condição menos restrita, em que para cada $A_{i}$ e $S_{2}^{*}$ verifica-se (2.13).

$$
\mathbb{P}\left(A_{i} \mid \bigcap_{j \in S_{2}^{*}} \bar{A}_{j}\right) \leq x_{i} \prod_{(i, j) \in E}\left(1-x_{j}\right)
$$

Assim, se para qualquer evento aleatório $A_{i}$, a probabilidade condicional dele, dado a não ocorrência mútua de qualquer conjunto de eventos aleatórios não adjacentes a ele no grafo de dependência é limitada superiormente por:

$$
x_{i} \prod_{(i, j) \in E}\left(1-x_{j}\right)
$$

temos verificadas as hipóteses do Lema Local de Lovász (2.1). 
Observação 2.3 A justificativa da observação anterior é devida ao fato de que na demonstração do Lema Local de Lovász (2.1), dado um evento aleatório $A_{i}$ qualquer, e os seguintes conjuntos:

- $S \subset\{1,2, \ldots, n\},|S|<n$ com $i \notin S$

- $S_{1}=\{j \in S ;(i, j) \in E\}$

- $S_{2}=\{j \in S ;(i, j) \notin E\}$

como $S$ é um subconjunto qualquer dos eventos aleatórios, temos que $S_{2}$ é qualquer subconjunto de eventos aleatórios que são mutuamente independentes de $A_{i}$. Ou seja, $S_{2}$ é qualquer subconjunto de vértices do grafo de dependência associado aos eventos aleatórios $A_{1}, A_{2}, \ldots, A_{n}$ que são não adjacentes a $A_{i}$.

Se considerarmos a hipótese menos restrita (2.13), temos satisfeita a desigualdade resultante da majoração feita para o numerador (2.10) e, por conseguinte, a afirmação expressa na desigualdade (2.5), que implica então o Lema Local de Lovász (2.1).

Observação 2.4 Quando $S=\left\{A_{1}, A_{2}, \ldots, A_{n}\right\} \backslash A_{i}$, temos que $S_{2}$ é o conjunto de todos os eventos aleatórios que são mutuamente independentes de $A_{i}$, neste caso, a hipótese (2.13), coincide com a hipótese (2.3).

\subsubsection{Condição suficiente para um limite inferior de $R(k, 3)$}

Considere agora o problema de se determinar uma condição suficiente para um limite inferior do número de Ramsey $R(k, 3)$ em que $k \neq 3$. Seja para tanto o conjunto $\Omega$ formado por todos os colorimentos $(\omega)$ de $K_{n}$, com a probabilidade de um elo ser azul $p$ e de ser vermelho $1-p$, e que cada elo é colorido independentemente dos demais. Assuma, por exemplo:

$$
\omega_{(i, j)}= \begin{cases}1 & \text { se o elo }(i, j) \text { for azul } \\ 0 & \text { se o elo }(i, j) \text { for vermelho }\end{cases}
$$

Sejam os conjuntos e eventos aleatórios definidos a seguir.

$\Omega:\{0,1\}^{\left(\begin{array}{l}n \\ 2\end{array}\right)}$ Representa o conjunto de todos os colorimentos de $K_{n}$ com as cores azul e vermelha. 
V: $\left\{v_{1}, v_{2}, \ldots, v_{n}\right\}$ Representa o conjunto de vértices de $K_{n}$.

$K_{i}:\left\{i_{1}, i_{2}, \ldots, i_{k}\right\} \subset \mathrm{V} ; \quad\left|K_{i}\right|=k \quad \forall i \in\left\{1,2, \ldots,\left(\begin{array}{l}n \\ k\end{array}\right)\right\} \quad$ Representa o i-ésimo subgrafo completo de ordem $k$, induzido pelos vértices de $K_{i}$ em $K_{n}$.

$T_{j}:\left\{j_{1}, j_{2}, j_{3}\right\} \subset \vee ; \quad\left|T_{j}\right|=3 \quad \forall j \in\left\{1,2, \ldots,\left(\begin{array}{l}n \\ 3\end{array}\right)\right\} \quad$ Representa o j-ésimo subgrafo completo de ordem 3 , induzido pelos vértices de $T_{l}$ em $K_{n}$.

$\mathrm{E}_{K_{i}}:\left\{\{u, v\} \subset\left\{i_{1}, i_{2}, \ldots, i_{k}\right\}: u<v\right\}$

Representa o conjunto dos elos de $K_{i} \quad \forall i \in\left\{1,2, \ldots,\left(\begin{array}{l}n \\ k\end{array}\right)\right\}$.

$\mathrm{E}_{T_{j}}:\left\{\{u, v\} \subset\left\{j_{1}, j_{2}, j_{3}\right\}: u<v\right\}$

Representa o conjunto dos elos de $T_{j} \quad \forall j \in\left\{1,2, \ldots,\left(\begin{array}{l}n \\ 3\end{array}\right)\right\}$.

$A_{K_{i}}:\left\{\forall\{u, v\} \subset\left\{i_{1}, i_{2}, \ldots, i_{k}\right\}: \omega_{u, v}=0\right\}$

Representa o evento aleatório de que todos os elos de $K_{i}$ são vermelhos.

$A_{T_{j}}:\left\{\forall\{u, v\} \subset\left\{j_{1}, j_{2}, j_{3}\right\}: \omega_{u, v}=1\right\}$

Representa o evento aleatório de que todos os elos de $T_{j}$ são azuis.

Do que foi definido acima, tem-se que para todo $i \in\left(\begin{array}{l}n \\ k\end{array}\right)$ e $j \in\left(\begin{array}{l}n \\ 3\end{array}\right)$ :

1. $\mathbb{P}\left(A_{K_{i}}\right):=\mathbb{P}\left(A_{K}\right)=(1-p)^{k}$

2. $\mathbb{P}\left(A_{T_{j}}\right):=\mathbb{P}\left(A_{T}\right)=(p)^{3}$

Seja o grafo de dependência (2.1) associado aos eventos $A_{K}$ e $A_{T}$, em que dois pontos (eventos) serão considerados adjacentes se apresentarem pelo menos um elo em comum.

Considere o grau de cada tipo de nó no grafo de dependência, ou seja, o número de elos nele incidente, e seja, $N_{x y}$, como o número de nós do tipo $x$ adjacente a um nó do tipo $y$.

1. $N_{K T} \leq\left(\begin{array}{l}n \\ k\end{array}\right)$ Pois existem no máximo $\left(\begin{array}{l}n \\ k\end{array}\right)$ subgrafos completos de ordem $k$ $\left(K_{k}\right)$, então, claramente não podemos ter mais que $\left(\begin{array}{l}n \\ k\end{array}\right) K_{k}$ adjacentes a $K_{3}$.

2. $N_{K K} \leq\left(\begin{array}{l}n \\ k\end{array}\right) \quad$ Justificativa análoga a do item anterior. 
3. $N_{T^{\prime} T}=3 \cdot(n-3)<3 \cdot n \quad$ Dado $T$, há $(n-3)$ outros pontos possíveis para formar um conjunto $T^{\prime}$ com um dos elos de $T$. Como há 3 possíveis escolhas de elo em $T$, segue o item 3 .

4. $N_{T K}=\left(\begin{array}{l}k \\ 2\end{array}\right) \cdot(n-k)$ Dado um nó do tipo $K$, ou seja, $K_{k}$, há $\left(\begin{array}{l}k \\ 2\end{array}\right)$ maneiras possíveis de se escolher o elo comum e depois há $(n-k)$ outras posíveis escolhas, independentes da primeira, para se formar $K_{3}$. Pelo princípio fundamental da contagem, segue o item 4.

$N_{T K}$ é majorado por:

$$
N_{T K}=\left(\begin{array}{l}
k \\
2
\end{array}\right) \cdot(n-k)<\frac{k^{2} \cdot n}{2}
$$

Podemos, então, usar o Lema Local de Lovász (2.1) para obter uma condição suficiente para um limite inferior de $R(k, 3)$. Para tanto, sejam $x, y$ e $p$ sujeito às seguintes restrições:

Impondo a $x$ e a $y$ a condição da primeira hipótese (2.2) temos:

$$
0 \leq x<1 \quad \text { e } \quad 0 \leq y<1
$$

Da segunda hipótese (2.3) temos:

$$
\begin{aligned}
p^{3} & \leq x \cdot(1-x)^{3 n} \cdot(1-y)^{\left(\begin{array}{l}
n \\
k
\end{array}\right)} \\
(1-p)^{\left(\begin{array}{l}
k \\
2
\end{array}\right)} & \leq y \cdot(1-x)^{\frac{k^{2} \cdot n}{2}} \cdot(1-y)^{\left(\begin{array}{l}
n \\
k
\end{array}\right)}
\end{aligned}
$$

Satisfeitas as duas restrições acima (2.14) e (2.15), temos então que $R(k, 3)>n$.

\subsection{O Lema Local de Lovász - caso simétrico.}

Sejam $A_{1}, A_{2}, \ldots, A_{n}$ eventos em um espaço de probabilidade qualquer, com probabilidades $p_{1}, p_{2}, \ldots, p_{n}$, respectivamente, e $p=\sup p_{i}$, com cada $A_{i}$ dependendo de no máximo $d$ outros eventos, sendo mutuamente independente dos demais.

Teorema 2.1 Se e $\times(d+1) \times p \leq 1$, então $\mathbb{P}\left(\bigcap_{i=1}^{n} \bar{A}_{i}\right)>0$.

Demonstração: 
- $d=0$

Então os eventos $A_{1}, A_{2}, \ldots, A_{n}$ são mutuamente independentes.

$$
\mathbb{P}\left(\bigcap_{i=1}^{n} \bar{A}_{i}\right)=\prod_{i=1}^{n} \mathbb{P}\left(\bar{A}_{i}\right)=\prod_{i=1}^{n}\left(1-\mathbb{P}\left(A_{i}\right)\right) \geq \prod_{i=1}^{n}(1-p)=(1-p)^{n}>0 .
$$

- $d \geq 1$

Seja $x_{i}=\frac{1}{d+1}$. Como $d \geq 1$, segue-se que $0 \leq x_{i}<1$, para todo $i \in\{1,2, \ldots, n\}$, a primeira hipótese (2.2) do Lema Local de Lovász (2.1) está satisfeita. Basta, então, verificar a segunda hipótese (2.3).

$$
\mathbb{P}\left(A_{i}\right) \leq x_{i} \prod_{(i, j) \in E}\left(1-x_{j}\right)
$$

Acontece que:

$$
\overbrace{\prod_{(i, j) \in E}\left(1-\frac{1}{d+1}\right)}^{I} \geq\left(1-\frac{1}{d+1}\right)^{d}>\frac{1}{e} .
$$

\section{Demonstração da primeira desigualdade:}

A primeira desigualdade é verdadeira porque (I) apresenta no máximo $d$ termos, pois para cada $A_{i}$, há no máximo $d$ outros eventos que não são mutuamente independentes com ele.

\section{Demonstração da segunda desigualdade:}

Temos que:

$$
\left(1-\frac{1}{d+1}\right)^{d}=\left(\frac{d}{d+1}\right)^{d}=\left(\frac{d+1}{d}\right)^{-d}=\left(1+\frac{1}{d}\right)^{-d}
$$

Acontece que a seqüência definida por:

$$
a_{d}=\left(1+\frac{1}{d}\right)^{d}
$$

é crescente e limitada; portanto apresenta limite.

$$
\lim _{d \rightarrow+\infty}\left(1+\frac{1}{d}\right)^{d}=e
$$


Segue-se portanto que para todo $d \geq 1$ :

$$
\left(1-\frac{1}{d+1}\right)^{d}>\frac{1}{e}
$$

Assim,

$$
\text { Se } e \times(d+1) \times p \leq 1 \text {, então } p \leq \frac{1}{d+1} \times \frac{1}{e}
$$

Como $P\left(A_{i}\right) \leq p$ para todo $i \in\{1,2, \ldots, n\}$, pois $p$ foi definido como $\sup P\left(A_{i}\right)=$ $\sup p_{i}$. De (2.17) e (2.16) temos:

$$
\begin{gathered}
\mathbb{P}\left(A_{i}\right) \leq p \leq \frac{1}{d+1} \times \frac{1}{e}<x_{i} \times\left(1-\frac{1}{d+1}\right)^{d} \leq x_{i} \times \prod_{(i, j) \in E}\left(1-\frac{1}{d+1}\right) \\
\Downarrow \\
\mathbb{P}\left(A_{i}\right) \leq x_{i} \times \prod_{(i, j) \in E}\left(1-x_{j}\right)
\end{gathered}
$$

Tem-se, portanto, que as duas hipóteses do lema estão satisfeitas a partir da condição $e \times(d+1) \times p \leq 1$. Logo esta condição é suficiente e segue-se o teorema (2.1)

\section{Observações do Lema Local de Lovász no caso simétrico:}

Observação 2.5 Considerando-se as hipóteses iniciais, (2.2) e (2.3), do Lema Local de Lovász (2.1), a condição $e \times(d+1) \times p \leq 1$, em que p é o sup $p_{i}$, pode ser substituída por e $\times(d+1) \times Q$, em que $Q$ é uma quantidade que necessariamente deve ser maior ou igual que o $\sup p_{i}$, pois, assim sendo, teríamos necessariamente que para todo $i \in\{1,2, \ldots, n\}$ :

$$
\sup p_{i} \leq Q \leq \frac{1}{d+1} \times \frac{1}{e} \leq x_{i} \times\left(1-\frac{1}{d+1}\right)^{d} \leq x_{i} \times \prod_{(i, j) \in E}\left(1-x_{j}\right)
$$

Observação 2.6 Da mesma forma, pode-se se considerar esta quantidade $Q$, para a hipótese menos restrita (2.13), como foi feito em (2.2), pois, nesse caso, bastaria que a probabilidade de qualquer $A_{i}$ dado o $S_{2}^{*}$ (2.12) teria que ser menor ou igual que $\frac{1}{d+1} \times \frac{1}{e}$, que é suficiente para,

$$
\mathbb{P}\left(A_{i} \mid \bigcap_{j \in S_{2}^{*}} \bar{A}_{j}\right) \leq x_{i} \prod_{(i, j) \in E}\left(1-x_{j}\right)
$$




\subsubsection{Aplicações}

Nesta subsecção serão apresentados resultados que podem ser construídos a partir do Lema Local de Lovász - caso simétrico (2.3). O Lema Local de Lovász aparece nestas aplicações como condição de suficiência para verificação de dois teoremas e de um limite inferior para o número de Ramsey na forma diagonal $R(k, k)$.

O teorema seguinte nos dá uma condição suficiente para a existência de um 2-colorimento em um hipergrafo, em função da estrutura de dependência entre seus hiperelos.

Definição 2.2 Hipergrafo K-Regular É o hipergrafo que a cada vértice apresenta $k$ hiperelos incidentes nele, ou seja, cada vértice pertence a $k$ hiperelos.

Teorema 2.2 Seja $\mathrm{H}=(\mathrm{V}, \mathrm{E})$ um hipergrafo no qual em todo hiperelo há pelo menos $k$ vértices e cada hiperelo de $\mathrm{H}$ intercepta no máximo d outros hiperelos. Nestas condições, se $e \cdot(d+1) \leq 2^{(k-1)}$, então $\mathrm{H}$ é 2-colorável.

Demonstração: Seja $A_{f}$ o evento aleatório de que o hiperelo $f$ é monocromático. Como para cada vértice podem ser escolhidas duas cores e por haver no mínimo $\mathrm{k}$ vértices em cada hiperelo, tem-se:

$$
\mathbb{P}\left(A_{f}\right)=2 \cdot \frac{1}{2^{|f|}} \leq \frac{1}{2^{k-1}} \quad \forall f \in \mathrm{E}
$$

Além disso, cada $A_{f}$ é mutuamente independente de todos os eventos aleatórios $A_{g}$, tais que os hiperelos $f$ e $g$ não se interceptam. Então, pelas condições do teorema, cada $A_{f}$ é dependente de no máximo $d$ outros eventos aleatótrios, sendo mutuamente independente dos demais. De (2.18), tem-se que:

$$
p=\sup P\left(A_{f}\right) \leq \frac{1}{2^{k-1}} \Rightarrow e \cdot(d+1) \cdot p \leq 1
$$

De (2.19) e (2.3) tem-se que há, portanto, uma probabilidade positiva associada ao evento aleatório de o hipergrafo não apresentar qualquer um de seus hiperelos monocromático, ou seja, de ser 2-colorável. 
Observação 2.7 A condição dada no teorema 2.2 para a existência de um 2-colorimento é mais geral que a dada no teorema 1.3, uma vez que aqui é estabelecida uma relação de dependência entre os hiperelos do hipergrafo.

Corolário 2.1 Se $k \geq 9$, todo hipergrafo k-uniforme e k-regular, é 2-colorável.

Demonstração: Segue do teorema 2.2, bastando observar os seguintes fatos:

- Cada hiperelo intercepta no máximo $k \cdot(k-1)$ outros hiperelos, pois como o hipergrafo é $k$-uniforme, cada hiperelo possui $k$ vértices e a cada um desses $k$ vértices pertencem a k-1 outros possíveis hiperelos, pois o hipergrafo também é $k$-regular.

- O evento aleatório $A_{f}=\{$ o hiperelo $f$ é monocromático $\}$, é tal que:

$$
P\left(A_{f}\right)=\frac{1}{2^{k-1}} \quad \forall f \in \mathrm{E} \quad \Rightarrow \quad \sup P\left(A_{f}\right)=\frac{1}{2^{k-1}}
$$

Assim, de (2.20) a condição de suficiência do Lema Local de Lovász - caso simétrico (2.3) se escreve:

$$
e \cdot(k \cdot(k-1)+1) \cdot \frac{1}{2^{k-1}} \leq 1 \quad \Leftrightarrow \quad e \cdot(k \cdot(k-1)+1) \leq 2^{k-1}
$$

A desiguladade acima (2.21) é verdadeira para todo $k \geq 9$, o que significa que todo hipergrafo em que cada hiperelo apresenta pelo menos 9 vértices e cada vértice tem grau igual a quantidade de hiperelos é 2-colorável.

O teorema seguinte nos dá uma cota superior para o número máximo de entradas iguais de uma matriz quadrada $\boldsymbol{A}$, a fim de que não se verifique a existência de repetições de entradas cujos os índices que as definem estejam associados a uma permutação $\pi$.

\section{Latin Transversals}

Seja $\boldsymbol{A}$ uma matriz quadrada de ordem $n$ em que cada $a_{i j}$ é um número inteiro. Seja $\pi$ uma permutação aleatória no conjunto $\{1,2, \ldots, n\}$ que define $n$ 
entradas em $\boldsymbol{A}$. Tal permutação é chamada de um Latin Tranversals de $\boldsymbol{A}$, se os valores das entradas $a_{i \pi(i)}$ são todos distintos para todo $i \in\{1,2, \ldots, n\}$.

Teorema 2.3 Suponha $k \leq \frac{n-1}{4 e}$ e que nenhum inteiro apareça em mais do que $k$ entradas de $\boldsymbol{A}$. Então $\boldsymbol{A}$ tem um Latin Tranversals.

A demonstração do teorema (2.3) será feita através do Lema Local de Lovász - caso simétrico (2.3) e, para tanto, será considerada a hipótese menos restrita (2.6). Há um Latin Transversals em $\boldsymbol{A}$, se, e somente se, todas as entradas definidas pelos elementos associados a alguma permutação forem distintos.

Sejam $\pi$ uma permutação aleatória de $(1,2, \ldots, n)$ e os seguintes eventos abaixo definidos:

$$
\begin{gathered}
T=\left\{\left(i, j, i^{\prime}, j^{\prime}\right): i<i^{\prime}, j \neq j^{\prime} \text { e } a_{i j}=a_{i^{\prime} j^{\prime}}\right\} \\
A_{i j i^{\prime} j^{\prime}}=\left\{\left(i, j, i^{\prime}, j^{\prime}\right) \in T: \pi(i)=j \text { e } \pi\left(i^{\prime}\right)=j^{\prime}\right\}
\end{gathered}
$$

- $T$ é o conjunto de 4-uplas cujos dois primeiros e dois últimos elementos definem entradas iguais na matriz $\boldsymbol{A}$, satisfazendo as restrições de as mesmas não pertencerem a uma mesma linha, pois $i<i^{\prime}$, e a uma mesma coluna, porque $j \neq j^{\prime}$.

- $A_{i j i^{\prime} j^{\prime}}$ corresponde ao evento aleatório em que para duas entradas iguais, ou seja, $a_{i j}=a_{i^{\prime} j^{\prime}}$, os elementos que definem cada uma das entradas estão associados a permutação aleatória. Assim, $A_{i j i^{\prime} j^{\prime}}$ ocorre, se e somente se, $a_{i j}=a_{i^{\prime} j^{\prime}}, \pi(i)=j$ e $\pi\left(i^{\prime}\right)=j^{\prime}$.

- O evento $\bar{A}_{i j i^{\prime} j^{\prime}}$ acontece sempre que $a_{i j}=a_{i^{\prime} j^{\prime}}$, pois $\left(i, j, i^{\prime}, j^{\prime}\right) \in T$, e pelo menos um dos eventos aleatórios ocorre: ou $\pi(i) \neq j$ ou $\pi\left(i^{\prime}\right) \neq j^{\prime}$. Assim, se ocorre o evento $\bar{A}_{i j i^{\prime} j^{\prime}}$ teremos, necessariamente, que as entradas definidas por cada um dos pares de elementos associados a permutação são distintas, pois se as entradas fossem iguais, teríamos a ocorrência simultânea de $A_{i j i^{\prime} j^{\prime}}$ e $\bar{A}_{i j i^{\prime} j^{\prime}}$ (absurdo !). 
associados a permutação aleatória. Assim, $A_{i j i^{\prime} j^{\prime}}$ ocorre, se e somente se, $a_{i j}=a_{i^{\prime} j^{\prime}}, \pi(i)=j$ e $\pi\left(i^{\prime}\right)=j^{\prime}$.

- O evento $\bar{A}_{i j i^{\prime} j^{\prime}}$ acontece sempre que $a_{i j}=a_{i^{\prime} j^{\prime}}$, pois $\left(i, j, i^{\prime}, j^{\prime}\right) \in T$, e pelo menos um dos eventos aleatórios ocorre: ou $\pi(i) \neq j$ ou $\pi\left(i^{\prime}\right) \neq j^{\prime}$. Assim, se ocorre o evento $\bar{A}_{i j i^{\prime} j^{\prime}}$ teremos, necessariamente, que as entradas definidas por cada um dos pares de elementos associados a permutação são distintas, pois se as entradas fossem iguais, teríamos a ocorrência simultânea de $A_{i j i^{\prime} j^{\prime}}$ e $\bar{A}_{i j i^{\prime} j^{\prime}}$ (absurdo !).

Exemplo 2.1 $O$ evento aleatório $A_{1122}$ ocorre, se e somente se:

$a_{11}=a_{22}, \pi(1)=1$ e $\pi(2)=2$.

Exemplo 2.2 $O$ evento aleatório $A_{i j i^{\prime} j^{\prime}}$ ocorre, se e somente se: $\{i, j\} \cap\left\{i^{\prime}, j^{\prime}\right\}=\varnothing$ e $a_{i j}=a_{i^{\prime} j^{\prime}}$

Afirmação 2.2 A condição necessária e suficiente de que A tenha um Latin Transversals é que:

$$
A=\bigcap_{T} \bar{A}_{i j i^{\prime} j^{\prime}} \neq \varnothing
$$

- O evento $A$ ocorre, se e somente se, todas as entradas da matriz $\boldsymbol{A}$ que forem iguais não sejam definidas a partir de elementos associados a uma permutação. $\mathrm{Ou}$, equivalentemente, se todas as entradas formadas a partir de elementos associados a uma permutação sejam distintas.

Sejam as duas seguintes proposições:

p : $\boldsymbol{A}$ tem um Latin Transversals

$\mathrm{q}: \bigcap_{T} \bar{A}_{i j i^{\prime} j^{\prime}} \neq \varnothing$

- $T=\varnothing$ 
Se $T=\varnothing$, então não existem duas entradas $a_{i j}$ e $a_{i^{\prime} j^{\prime}}$ de tal forma que $a_{i j}=a_{i^{\prime} j^{\prime}}$. Logo, a matriz apresenta todas as entradas distintas e, por conseguinte, aquelas que estão também associadas a permutações aleatórias. Logo, há um Latin Transversals.

- $T \neq \varnothing \quad$ A condição é necessária $(p \Rightarrow q)$

Temos que mostrar que $\mathrm{p} \Rightarrow \mathrm{q}$.

Se há um Latin Transversals, então segue da definição, que as entradas de $\boldsymbol{A}=\left(a_{i j}\right)$, em que $\pi(i)=j$, são distintas para todo $i \in\{1,2, \ldots, n\}$. Logo, as entradas de elementos associados a uma permutação são distintas e portanto $A$ ocorre.

- $T \neq \varnothing \quad$ A condição é suficiente $(q \Rightarrow p)$

Temos que mostrar que $\mathrm{q} \Rightarrow \mathrm{p}$.

Se o evento aleatório $A$ ocorre, então todas as entradas que são iguais não estão associadas a elementos da permutação. Logo, as entradas de elementos associados a uma permutação são distintas e portanto $A$ ocorre, ou seja, há um Latin Transversals.

Seja $G=(V, E)$ o Grafo de Dependência associado aos pontos de $T$, de forma que dois pontos $t_{1}=\left(i, j, i^{\prime}, j^{\prime}\right)$ e $t_{2}=\left(p, q, p^{\prime}, q^{\prime}\right)$ são considerados adjacentes, ou seja, há um elo os unindo, se:

$$
\left\{i, i^{\prime}\right\} \cap\left\{p, p^{\prime}\right\} \neq \varnothing
$$

Esta condição implica que quaisquer das duas entradas de $t_{1}$ e $t_{2}$ não ocupam a mesma linha.

$$
\left\{j, j^{\prime}\right\} \cap\left\{q, q^{\prime}\right\} \neq \varnothing
$$

De maneira análoga, acima temos a condição de que quaisquer das duas entradas de $t_{1}$ e $t_{2}$ não ocupam a mesma coluna.

Assim, os dois pontos $t_{1}$ e $t_{2}$ não serão considerados adjacentes se as quatro células $(i, j),\left(i^{\prime}, j^{\prime}\right),(p, q)$ e $\left(p^{\prime}, q^{\prime}\right)$ ocupam quatro linhas e quatro colunas diferentes de $\boldsymbol{A}$, ou então, serão considerados adjacentes se qualquer uma das quatro células fica 
na mesma coluna ou na mesma linha.

Cálculo do grau máximo (d) de G : Dado um ponto $\left(i, j, i^{\prime}, j^{\prime}\right) \in T$, há $4 \cdot n$ escolhas para $(p, q)$ tal que $a_{p q}$ seja qualquer uma das entradas das duas linhas $p \in\left(i, i^{\prime}\right)$ ou duas colunas $q \in\left(j, j^{\prime}\right)$ ocupadas por $a_{i j}$ e $a_{i^{\prime} j^{\prime}}$. Para cada uma dessas $4 \cdot n$ escolhas de $(p, q)$, há no máximo $k-1$ entradas $a_{p^{\prime} q^{\prime}}$ de $\boldsymbol{A}$ tais que $a_{p q}=a_{p^{\prime} q^{\prime}}$ e $(p, q) \neq\left(p^{\prime}, q^{\prime}\right)$. Portanto, cada vértice em G é adjacente a no máximo $d=4 \cdot n \cdot(k-1)$ outros vértices. Logo, o grau máximo de $\mathrm{G}$ é menor que $4 \cdot n \cdot k$.

$\operatorname{Seja}\left(i, j, i^{\prime}, j^{\prime}\right) \in T$ e $S=\left\{\left(p, q, p^{\prime}, q^{\prime}\right):\left(\left(p, q, p^{\prime}, q^{\prime}\right),\left(i, j, i^{\prime}, j^{\prime}\right)\right) \notin \mathrm{E}\right\}$.

- $S$ é o subconjunto de pontos pertencentes a $T$ e que não são adjacentes a $\left(i, j, i^{\prime}, j^{\prime}\right)$ no grafo $\mathrm{G}$.

- $S$ representa o conjunto de entradas iguais de $\boldsymbol{A}$ que não estão na mesma linha ou na mesma coluna das entradas $a_{i j}$ e $a_{i^{\prime} j^{\prime}}$.

Temos como hipótese do teorema (2.3) que:

$$
\begin{gathered}
k \leq \frac{n-1}{4 \cdot e} \\
\Downarrow \\
\frac{4 \cdot e \cdot k}{n-1} \leq 1
\end{gathered}
$$

Seja $d$ o grau máximo de G. Temos:

$$
\begin{gathered}
d<4 \cdot n \cdot k \\
\Downarrow \\
(d+1) \leq 4 \cdot n \cdot k
\end{gathered}
$$

Das deigualdades acima, tem-se que:

$$
\frac{4 \cdot e \cdot k}{n-1} \leq 1
$$




$$
\begin{gathered}
\Downarrow \\
\frac{4 \cdot n \cdot e \cdot k}{n \cdot(n-1)} \leq 1
\end{gathered}
$$

De (2.26) e (2.25) tem-se:

$$
e \cdot(d+1) \cdot \frac{1}{n \cdot(n-1)} \leq 1
$$

O grafo associado ao conjunto $T$ apresenta, para cada vértice de $T$, no máximo $d=4 \cdot n \cdot(k-1)$ outros vértices adjacentes.

Assim, se cada vértice é visto como sendo um evento aleatório, estamos nas condições do Lema Local de Lovász - caso simétrico (2.3).

Seja a quantidade:

$$
\begin{array}{r}
Q=\frac{1}{n \cdot(n-1)} \\
e \cdot(d+1) \cdot \frac{1}{n \cdot(n-1)} \leq 1 \\
\Downarrow \\
Q \leq \frac{1}{e \cdot(d+1)}
\end{array}
$$

Assim, considerando a observação a respeito da hipótese menos restrita no caso simétrico (2.6), é suficiente mostrar que para a ocorrência de um Latin Transversals.

$$
\mathbb{P}\left(A_{i j i^{\prime} j^{\prime}} \mid \bigcap_{j \in S} \bar{A}_{p q p^{\prime} q^{\prime}}\right) \leq \frac{1}{n \cdot(n-1)}
$$

Definição 2.3 (Boa Permutação) Dado o conjunto $T$, um ponto $\left(i, j, i^{\prime}, j^{\prime}\right) \in$ $T$ e $S=\left\{\left(p, q, p^{\prime}, q^{\prime}\right):\left(\left(p, q, p^{\prime}, q^{\prime}\right),\left(i, j, i^{\prime}, j^{\prime}\right)\right) \notin \mathrm{E}\right\}$. A permutação $\pi e ́$ boa se ela satisfaz:

$$
\bigcap_{S} \bar{A}_{p q p^{\prime} q^{\prime}}
$$


Como $\pi^{*}(1)=i, \pi^{*}(2)=j$, se $\pi^{*}$ for uma boa permutação, implicaria que $\pi^{*} \in S_{i j}$. Para $i \in\{1,2\}$, seja $v_{i}$, tal que $v_{i} \notin\{1,2\}$

Seja o conjunto

$$
V=\left(v_{1}, \pi\left(v_{1}\right), v_{2}, \pi\left(v_{2}\right)\right)
$$

$V$ representa o conjunto das entradas de $\boldsymbol{A}$ em que $a_{v_{1} \pi\left(v_{1}\right)} \neq a_{v_{2} \pi\left(v_{2}\right)}$. Como $v_{1} \neq v_{2}$, implica $\pi\left(v_{1}\right) \neq \pi\left(v_{2}\right)$, temos, necessariamente, que as entradas em $\boldsymbol{A}$ associadas a cada um dos pontos de $V$, cada ponto define duas entradas, ocupam linhas e colunas distintas, ou seja, são não adjacentes.

Como o conjunto dos valores associados associados por $\pi^{*}$ é o mesmo de $\pi$, temos que todas as entradas associadas aos pontos não adjacentes a $(1, i, 2, j)$ na nova permutação são distintas.

- $\{1,2\} \cap\{i, j\} \neq \varnothing$.

Considere, por exemplo, $s_{12}$ e $s_{13}$. Logo, existe $x \in\{1,2, \ldots, n\} ; \pi(x) \notin\{1,2\}$. Considere, sem perda de generalidade, $\pi(x)=3$

$$
\pi^{*}(v)=\left\{\begin{array}{ccl}
1 & \text { se } & v=1 \\
3 & \text { se } & v=2 \\
2 & \text { se } & v=x \\
\pi(v) & \text { se } & v \neq\{1,2, x\}
\end{array}\right.
$$

Pelas mesmas razões consideradas anteriormente, a nova transformação $\pi^{*}$ faz com que todas as entradas de $\boldsymbol{A}$ associada aos pontos não adjacentes a $(1,1,2,3)$ sejam distintas.

Para mostrar a existência de um Latin Transversals vimos que era suficiente que:

$$
\mathbb{P}\left(A_{i j i^{\prime} j^{\prime}} \mid \bigcap_{j \in S} \bar{A}_{p q p^{\prime} q^{\prime}}\right) \leq \frac{1}{n \cdot(n-1)}
$$

Proposição 2.1

$$
\mathbb{P}\left(A_{i j i^{\prime} j^{\prime}} \mid \bigcap_{j \in S} \bar{A}_{p q p^{\prime} q^{\prime}}\right) \leq \frac{1}{n \cdot(n-1)}
$$


O evento aletório $\left\{A_{i j i^{\prime} j^{\prime}} \mid \bigcap_{j \in S} \bar{A}_{p q p^{\prime} q^{\prime}}\right\}$ é o conjunto das boas permutações tais que $\pi(i)=j$ e $\pi\left(i^{\prime}\right)=j^{\prime}$

Assim,

$$
\mathbb{P}\left(A_{i j i^{\prime} j^{\prime}} \mid \bigcap_{j \in S} \bar{A}_{p q p^{\prime} q^{\prime}}\right)=\mathbb{P}\left(A_{i j i^{\prime} j^{\prime}} \text { ser boa permutação }\right)
$$

Acontece que:

$$
\mathbb{P}\left(A_{i j i^{\prime} j^{\prime}} \text { ser boa permutação }\right)=\mathbb{P}\left(A_{1122} \text { ser boa permutação }\right)=\frac{s_{12}}{\sum_{i \neq j} s_{i j}}
$$

mas como $s_{12} \leq s_{i j} \forall i \neq j$

$$
\frac{s_{12}}{\sum_{i \neq j} s_{i j}} \leq \frac{s_{12}}{\sum_{i \neq j} s_{12}}=\frac{s_{12}}{n \cdot(n-1) s_{12}}=\frac{1}{n \cdot(n-1)}
$$

No teorema 1.1 foi dado um limite inferior para o número de Ramsey. A partir da condição dada no teorema, foi obtido no apêndice [A1] um outro limite, superior ao primeiro, e, portanto, melhor. O Lema Local de Lovász nos fornece uma condição de obtermos um limite inferior para $R(k, k)$ superior aos outros dois primeiros limites já citados.

\section{Condição suficiente para um limite inferior do número de Ramsey $R(k, k)$}

Considere um grafo completo de ordem $n\left(K_{n}\right)$ e que cada um dos seus elos sejam coloridos independentemente um dos outros e com mesma probabilidade de ser azul ou vermelho.

$$
\omega_{(i, j)}= \begin{cases}1 & \text { se o elo }(i, j) \text { for azul. } \\ 0 & \text { se o elo }(i, j) \text { for vermelho }\end{cases}
$$

O valor de $R(k, k)$ é então o menor valor de $n$ para o qual em qualquer colorimento de $K_{n}$ há pelo menos um subgrafo completo de ordem $k\left(K_{k}\right)$ monocromático. Seja $A_{i}=\{$ o i-ésimo subgrafo completo de ordem $k$ é monocromático $\}$. Considere 
$A_{i}$ como sendo independente de $A_{j}$, se ambos não apresentarem qualquer elo em comum, ou seja, se o conjunto dos vértices de $A_{i}$ e $A_{j}$ não apresentarem dois ou mais elementos em sua interseç̧ão. Então:

1. $\mathbb{P}\left(\omega_{i j}=1\right)=\mathbb{P}\left(\omega_{i j}=0\right)=\frac{1}{2}$

2. $\mathbb{P}\left(A_{i}\right)=2 \cdot \frac{1}{2^{\left(\begin{array}{c}k \\ 2\end{array}\right)}}=2^{1-\left(\begin{array}{c}k \\ 2\end{array}\right)}$

3. O grau máximo de cada $A_{i}$ no grafo de dependência é $d \leq\left(\begin{array}{l}k \\ 2\end{array}\right) \cdot\left(\begin{array}{c}n \\ k-2\end{array}\right)$

Tem-se, portanto, da secção (2.3), que a condição abaixo é suficiente para que não exista nenhum subgrafo completo de ordem $k$ :

$$
e \cdot\left(\left(\begin{array}{l}
k \\
2
\end{array}\right) \cdot\left(\begin{array}{c}
n \\
k-2
\end{array}\right)+1\right) \cdot 2^{1-\left(\begin{array}{l}
k \\
2
\end{array}\right)}<1
$$

Temos em Alon [1], que o valor de $n$ que satisfaz a desigualdade acima é:

$$
R(k, k)>\frac{2 \cdot k}{e \cdot \sqrt{2}} \cdot 2^{\frac{k}{2}}
$$

Observação 2.8 Como há uma simetria na estrutura de dependência entre os eventos considerados, estamos portanto nas condições de uso da versão do Lema no caso simétrico. 


\section{Apêndice A}

\section{[A1] Limite inferior para $R(k, k)$}

Pode-se apresentar um limite inferior maior para o número de Ramsey na forma diagonal - $R(k, k)$ superior ao obtido no teorema (1.1). Esta mesma condição também poderia ser derivada a partir do Método do Primeiro Momento de acordo com o que foi descrito no primeiro parágrafo da secção 1.3, página 11.

$$
\mathbb{P}\left(\bigcup_{i=1}^{n} A_{i}\right) \leq\left(\begin{array}{l}
n \\
k
\end{array}\right) \cdot 2^{1-\left(\begin{array}{l}
k \\
2
\end{array}\right)<1}
$$

Seja a função $f(n, k)$ definida por:

$$
f(n, k)=\left(\begin{array}{l}
n \\
k
\end{array}\right) \cdot 2^{1-\left(\begin{array}{c}
k \\
2
\end{array}\right)}
$$

Um limite inferior pode ser estabelecido para $R(k, k)$ desde que (A) esteja satisfeita. Fixado $k, f(n, k)$ passa a ser uma função crescente de $n$. Seja então $n_{0}=n_{0}(k)$ tal que $f\left(n_{0}, k\right)<1$.

Proposição A.1 $\frac{1}{k !} \leq\left(\frac{e}{k}\right)^{k} \cdot \frac{1}{e}$

Demonstração: considere o gráfico de $y=\ln x$, e para cada $j \geq 2$, intervalos da forma $[j-1, j]$. A área compreendida pelo gráfico da função em cada intervalo é menor que a área do retângulo cuja base fica no respectivo intervalo. Assim:

$$
\int_{j-1}^{j} \ln x d x \leq \ln j \cdot[j-(j-1)]=\ln j
$$

Logo,

$$
\ln k !=\sum_{j=2}^{k} \ln j \geq \sum_{j=2}^{k} \int_{j-1}^{j} \ln x d x=\int_{1}^{k} \ln x d x=k \cdot \ln k-k+1
$$


O que implica que:

$$
k ! \geq k^{k} \cdot e^{1-k}=\left(\frac{k}{e}\right)^{k} e \Rightarrow \frac{1}{k !} \leq\left(\frac{e}{k}\right)^{k} \cdot \frac{1}{e}
$$

Temos também a seguinte majoração.

$$
\left(\begin{array}{l}
n \\
k
\end{array}\right)=\frac{n \cdot(n-1) \times \ldots \times(n-k+1)}{k !}<\frac{n^{k}}{k !}
$$

De (A.1) e (A.2 ) tem-se que:

$$
\left(\begin{array}{l}
n \\
k
\end{array}\right)<\left(\frac{n \cdot e}{k}\right)^{k} \cdot \frac{1}{e}
$$

Temos, portanto, de (A.3) que:

$$
\left(\begin{array}{l}
n \\
k
\end{array}\right) \cdot 2^{1-\left(\begin{array}{l}
k \\
2
\end{array}\right)}<\left(\frac{n \cdot e}{k}\right)^{k} \cdot \frac{1}{e} \cdot 2^{1-\left(\begin{array}{l}
k \\
2
\end{array}\right)}<\left(\frac{n \cdot e}{k}\right)^{k} \cdot 2^{-\left(\begin{array}{l}
k \\
2
\end{array}\right)}
$$

Seja então a função $g(n, k)$ assim definida:

$$
g(n, k)=\left(\frac{n \cdot e}{k}\right)^{k} \cdot 2^{-\left(\begin{array}{c}
k \\
2
\end{array}\right)}
$$

De (A.5) e (A.4) tem-se que $f(n, k)<g(n, k)$. Logo, se exibirmos um $n_{0}=n_{0}(k)$ tal que $g\left(n_{0}, k\right)=1$, então necessariamente $f\left(n_{0}, k\right)<1 \quad \forall n \leq n_{0}$, que é equivalente a dizer que $R(k, k)>n_{0}$. Temos, portanto, que o valor de $n_{0}$ que satisfaz $g\left(n_{0}, k\right)=1$ é dado por:

$$
\left(\frac{n_{0} \cdot e}{k}\right)^{k} \cdot 2^{-\left(\begin{array}{c}
k \\
2
\end{array}\right)}=1 \quad \Leftrightarrow \quad\left(\frac{n_{0} \cdot e}{k}\right)^{k}=2^{\left(\begin{array}{c}
k \\
2
\end{array}\right)} \quad \Leftrightarrow \quad n_{0}=\frac{k}{e \cdot \sqrt{2}} \cdot 2^{\frac{k}{2}}
$$




\section{[A2] Outras Representações do Lema Local de Lovász}

O Lema Local de Lovász pode assumir outras versões. Contudo, todas elas como casos particulares da versão mais geral do Lema (2.1). Tais outras representações apresentam-se significativamente mais importantes quando comparadas a versão simétrica do Lema (2.3), pois neste caso temos condições gerais de majoração para $p=\sup P\left(A_{i}\right)$ e $d$.

\section{- Lema Local na Forma Assimétrica}

Sejam $A_{1}, A_{2}, \ldots, A_{n}$ eventos e $\mathrm{G}_{d}=(\mathrm{V}, \mathrm{E})$ o grafo de dependência associado a eles. Para cada $i \in\{1,2, \ldots, n\}, \mathrm{D}_{i}=\{j:(i, j) \in \mathrm{E}\}$. Nestas condições, se:

1. $\mathbb{P}\left(A_{i}\right) \leq \frac{1}{4}$

2. $\sum_{j \in \mathrm{D}_{i}} \mathbb{P}\left(A_{j}\right) \leq \frac{1}{4}$

Então com probabilidade positiva nenhum $\operatorname{dos} A_{i}$ ocorre.

\section{Demonstração:}

Considere $x_{i}$ como descrito em (2.2). Seja $x_{i}=2 \cdot \mathbb{P}\left(A_{i}\right) \Rightarrow x_{i} \leq \frac{1}{2}$. Claramente, $1-x \geq\left(\frac{1}{4}\right)^{x}$ para $x \in\left(0, \frac{1}{2}\right)$. Sejam então as duas seguintes condições:

1. $x_{i}=2 \cdot \mathbb{P}\left(A_{i}\right)$

2. $\alpha=2 \cdot \ln 2$

3. $(1-x) \geq \exp (-\alpha \cdot x)$

Temos então que a segunda hipótese (2.3) do Lema Local de Lovász (2.1) também é satisfeita, pois:

$$
\begin{aligned}
x_{i} \cdot \prod_{j \in \mathrm{D}_{i}}\left(1-x_{j}\right) & \geq x_{i} \cdot \prod_{j \in \mathrm{D}_{i}} \exp \left(-\alpha \cdot x_{j}\right)= \\
2 \cdot \mathbb{P}\left(A_{i}\right) \exp \left(-\alpha \cdot \sum_{j \in \mathrm{D}_{i}} 2 \cdot \mathbb{P}\left(A_{j}\right)\right) & \geq 2 \cdot \mathbb{P}\left(A_{i}\right) \cdot \exp \left(-\frac{\alpha}{2}\right)=\mathbb{P}\left(A_{i}\right) \\
& \Leftrightarrow \\
\mathbb{P}\left(A_{i}\right) & \leq x_{i} \cdot \prod_{j \in \mathrm{D}_{i}}\left(1-x_{j}\right)
\end{aligned}
$$




\section{- Lema Local na Forma Ponderada}

Sejam $A_{1}, A_{2}, \ldots, A_{n}$ eventos e $\mathrm{G}_{d}=(\mathrm{V}, \mathrm{E})$ o grafo de dependência associado a eles. Para cada $i \in\{1,2, \ldots, n\}, \mathrm{D}_{i}=\{j:(i, j) \in \mathrm{E}\}$. Nestas condições, se existirem inteiros $t_{1}, t_{2}, \ldots, t_{n}$ e $p \in\left[0, \frac{1}{4}\right]$ e as duas condições abaixo estiverem satisfeitas:

1. $\mathbb{P}\left(A_{i}\right) \leq p^{t_{i}}$

2. $\sum_{j \in \mathrm{D}_{i}}(2 p)^{t_{i}} \leq \frac{t_{i}}{2}$

Então com probabilidade positiva nenhum dos $A_{i}$ ocorre.

\section{Demonstração:}

Considere o mesmo conjunto de transformações feito anteriormente. Temos que verificar que a partir delas e usando as hipóteses do Lema na forma acima especeficada, nos dá suficiência para a verificação das hipóteses quando o Lema está na forma geral (2.1).

- $x_{i}=2 \cdot \mathbb{P}\left(A_{i}\right)$

- $\alpha=2 \cdot \ln 2$

- $(1-x) \geq \exp (-\alpha \cdot x)$

Como $p \in\left[0, \frac{1}{4}\right]$ e $t_{i} \geq 1$, temos que $x_{i}=2 \cdot \mathbb{P}\left(A_{i}\right) \in\left[0, \frac{1}{2}\right]$ e portanto a hipótese (2.2) do Lema na forma geral está satisfeita.

Da mesma forma como foi feito na prova anterior, seja a desigualdade $(1-x) \geq$ $\exp (-\alpha \cdot x)$ que vale nas condições de $\alpha=2 \cdot \ln 2$ e $x \in\left[0, \frac{1}{2}\right]$. Tem-se:

$$
\begin{aligned}
x_{i} \cdot \prod_{j \in \mathrm{D}_{i}}\left(1-x_{j}\right) & =(2 \cdot p)^{t_{i}} \cdot \prod_{j \in \mathrm{D}_{i}}\left(1-(2 \cdot p)^{t_{i}}\right) \geq \\
(2 \cdot p)^{t_{i}} \cdot \exp \left(-\alpha \cdot \sum_{j \in \mathrm{D}_{i}}(2 \cdot p)^{t_{j}}\right) & \geq(2 \cdot p)^{t_{i}} \cdot \exp \left(-\alpha \cdot \frac{t_{i}}{2}\right)= \\
(p)^{t_{i}} & \geq \mathbb{P}\left(A_{i}\right) \\
& \Leftrightarrow \\
\mathbb{P}\left(A_{i}\right) & \leq x_{i} \cdot \prod_{j \in \mathrm{D}_{i}}\left(1-x_{j}\right)
\end{aligned}
$$




\section{Referências Bibliográficas}

[1] Alon, N. e Spencer, J. H. The Probabilistic Method. 2nd. ed., John Wiley and Sons, New York, 1991.

[2] ERDös, P. (1947). Some remarks on the theory of graphs, Bull. Amer. Math. Soc. 53: 292-294.

[3] Erdös, P. e Spencer, J. H. Probabilistic methods in combinatorics. Academic Press, New York, 1974.

[4] ERdÖs, P. e Spencer, J. H. (1991). Lopsided Lovász local lemma and latin transversals, Discrete Apl. Math. 30: 151-154.

[5] Harary, F. Graph Theory. 3rd. ed., Adisson-Wesley, Massachusetts, 1972.

[6] Reed, B. E Molloy, M. Graph Colouring and the Probabilistic Method. Springer-Verlag, Berlin, 2002.

[7] Spencer, J. H. Ten Lectures On the Probabilistic Method. 2nd. ed., SIAM, Philadelphia, 1994.

[8] Spencer, J. H. (1977). Assimptotic lower bounds for Ramsey functions, Disc. Math. 20: 69-76.

[9] Szele, T. (1943). Kombinatorikai vizsgálatok az irányiott teljes gráffal kapcsolatban, Mat. Fiz. Lapok 50: 223-256.

[10] Tutre, W. T. Graph theory. Addison-Wesley, Menlo Park, 1984. 